\title{
Kinematics of the jet in M 87 on scales of 100-1000 Schwarzschild radii
}

\author{
F. Mertens ${ }^{1,2}$, A. P. Lobanov ${ }^{1,3}$, R. C. Walker ${ }^{4}$, and P. E. Hardee ${ }^{5}$ \\ 1 Max-Planck-Institut für Radioastronomie, Auf dem Hugel 69, 53121 Bonn, Germany \\ e-mail: florent .mertens@gmail.com \\ 2 Kapteyn Astronomical Institute, PO Box 800, 9700 AV Groningen, The Netherlands \\ 3 Institut für Experimentalphysik, Universität Hamburg, Luruper Chaussee 149, 22761 Hamburg, Germany \\ ${ }^{4}$ National Radio Astronomy Observatory, Socorro, NM 87801, USA \\ 5 Department of Physics \& Astronomy, The University of Alabama, Tuscaloosa, AL 35487, USA
}

Received 30 April 2016 / Accepted 12 August 2016

\begin{abstract}
Context. Very long baseline interferometry (VLBI) imaging of radio emission from extragalactic jets provides a unique probe of physical mechanisms governing the launching, acceleration, and collimation of relativistic outflows.

Aims. VLBI imaging of the jet in the nearby active galaxy M 87 enables morphological and kinematic studies to be done on linear scales down to $\sim 100$ Schwarzschild radii $\left(R_{\mathrm{s}}\right)$.

Methods. The two-dimensional structure and kinematics of the jet in M 87 (NGC 4486) have been studied by applying the waveletbased image segmentation and evaluation (WISE) method to 11 images obtained from multi-epoch Very Long Baseline Array (VLBA) observations made in January-August 2007 at $43 \mathrm{GHz}(\lambda=7 \mathrm{~mm})$.

Results. The WISE analysis recovers a detailed two-dimensional velocity field in the jet in M 87 at sub-parsec scales. The observed evolution of the flow velocity with distance from the jet base can be explained in the framework of MHD jet acceleration and Poynting flux conversion. A linear acceleration regime is observed up to $z_{\mathrm{obs}} \sim 2$ mas. The acceleration is reduced at larger scales, which is consistent with saturation of Poynting flux conversion. Stacked cross correlation analysis of the images reveals a pronounced stratification of the flow. The flow consists of a slow, mildly relativistic layer (moving at $\beta \sim 0.5 c$ ), associated either with instability pattern speed or an outer wind, and a fast, accelerating stream line (with $\beta \sim 0.92$, corresponding to a bulk Lorentz factor $\gamma \sim 2.5$ ). A systematic difference of the apparent speeds in the northern and southern limbs of the jet is detected, providing evidence for jet rotation. The angular velocity of the magnetic field line associated with this rotation suggests that the jet in M 87 is launched in the inner part of the disk, at a distance $r_{0} \sim 5 R_{\mathrm{s}}$ from the central engine.

Conclusions. The combined results of the analysis imply that MHD acceleration and conversion of Poynting flux to kinetic energy play the dominant roles in collimation and acceleration of the flow in M 87.
\end{abstract}

Key words. galaxies: active - galaxies: individual: M 87 - galaxies: jets - magnetohydrodynamics (MHD)

\section{Introduction}

M 87 (Virgo A, NGC 4486, 3C 274) is a giant elliptical galaxy. Its proximity $(D=16.7 \mathrm{Mpc}$; Mei et al. 2007) combined with the large mass of its central black hole $\left(M_{\mathrm{BH}} \simeq 6.1 \times 10^{9} M_{\odot}\right.$; Gebhardt et al. 2011) make M87 one of the primary sources to probe jet formation and acceleration down to smallest linear scales (with 1 mas $\approx 0.08 \mathrm{pc} \sim 140$ Schwarzschild radii $\left(R_{\mathrm{s}}\right)$ ).

The kiloparsec-scale structure of the jet in M 87 has been extensively studied in the radio regime using the Very Large Array (VLA; Owen et al. 1989), the optical regime using the Hubble Space Telescope (HST; Biretta et al. 1995), and the X-ray regime using the Chandra space telescope (Marshall et al. 2002). These observations have revealed an edge brightened conical jet with an apparent opening angle $\Theta_{\mathrm{obs}} \sim 3.2^{\circ}$ (in this paper, "opening angle" refers to half the full opening angle). The jet manifests several bright knots, with a particularly strong feature HST-1 located at a projected distance of $z_{\mathrm{obs}} \sim 0.84^{\prime \prime}$. The jet expends uniformly up to knot $\mathrm{A}$ at a distance $z_{\mathrm{obs}} \sim 12^{\prime \prime}$. Analysis of multi-epoch observations has yielded detections of superluminal speeds of up to $\beta_{\text {app }} \sim 6 c$ in the optical (Biretta et al. 1999) and $\beta_{\text {app }} \sim 4 c$ in the radio (Biretta et al. 1995; Cheung et al. 2007; Giroletti et al. 2012) at the location of HST-1, with evidence of deceleration downstream from this point. Several components with subluminal speed $\left(\beta_{\text {app }} \sim 0.5 c\right)$ were also detected all along the jet (Biretta et al. 1999; Meyer et al. 2013), concurrently with the detections of superluminal features made at similar distances.

Both the radio and optical maps exhibit bright filaments, twisted into an apparent double-helical pattern. This has been successfully modeled as a consequence of Kelvin Helmholtz (K-H) instability developing in the jet (Lobanov et al. 2003; Hardee \& Eilek 2011), with the resulting expected pattern speed of $\approx 0.5 c$ that can explain the subluminal features found in the jet. In the optical regime, jet structure is dominated by knots, while the filaments are more pronounced in the radio images. This difference has been interpreted in the framework of a stratified jet by Perlman et al. (1999), in which the high energy plasma particles emitting optical synchrotron radiation originate from regions close to the spine of the flow, while the lower energy radio emitting particles are concentrated in the outer layers of the flow.

The physical nature of the inner jet in M 87 has been the subject of a number of studies (cf., Biretta \& Junor 1995, for 
a review). Recently, multi-frequency phase-referencing VLBI observations (Hada et al. 2011) were used to locate the central engine at a projected distance of only about $40 R_{\mathrm{S}}$ upstream from the base (or the core) of the radio jet observed at $43 \mathrm{GHz}$. Jet expansion can be described with a parabolic profile (Asada \& Nakamura 2012), indicating that the jet is collimated by magnetohydrodynamic (MHD) processes (Meier et al. 2001). The transition to a conical jet was found to occur at a projected distance of $\sim 350$ mas from the core.

Proper motions detected in the jet in M 87 at parsec scales have been the subject of an intensive debate (cf., Biretta \& Junor 1995; Kovalev et al. 2007; Ly et al. 2007; Walker et al. 2009). The jet has been observed at $2 \mathrm{~cm}$ wavelengths as part of the MOJAVE project, with a reported slow subluminal speed ( $\beta_{\text {app }} \sim 0.01 c$; Kovalev et al. 2007) which seems to be in contradiction with proper motions of up to $\beta_{\text {app }} \sim 4-6 c$ observed at HST-1 (Biretta et al. 1999). Recently, VLBI observations at $1.6 \mathrm{GHz}$ (Asada et al. 2014) have been used to claim a detection of gradual acceleration of the jet between 100 mas and 900 mas, suggesting a link between the subluminal acceleration found in the first 20 mas of the jet, and the relativistic speed at HST-1. A major issue persists in this picture: assuming that the jet is intrinsically bidirectional and symmetric, detection of a subluminal speed would imply a jet to counter-jet intensity ratio near unity, while the jet in M 87 is essentially one sided. The presence of a counter-jet is suggested from $15 \mathrm{GHz}$, $43 \mathrm{GHz}$, and $86 \mathrm{GHz}$ VLBI observations (Kovalev et al. 2007; Ly et al. 2007; Hada et al. 2016). However, the counter-jet emission is only about 2 mas in extent, and jet to counter-jet ratios of 5-20 are reported. These findings support an alternative scenario in which the ultra-slow speed of $\approx 0.01 c$ is either a pattern speed or a slow wind from an outer sheath (Kovalev et al. 2007). This conclusion is further strengthened by the observed limbbrightened morphology of the jet. Sparsity of the MOJAVE time sampling is a likely reason for the non-detection of superluminal speeds in M 87. The MOJAVE observations of M 87 are sparse, with an average time interval of about 200 days between successive epochs, resulting in a maximum reliably detectable speed of about $1 c$ (taken as a displacement of about the beam size between consecutive epochs). While Kovalev et al. (2007) could not identify any fast component, Ly et al. (2007) reported possible features with speeds between $0.25 c$ and $0.4 c$ at a distance of 3 mas from the core, with the detection obtained from only two epochs.

In an attempt to finally assess the true bulk speed of the jet at sub-parsec scales, systematic VLBA ${ }^{1}$ observations were performed by Walker et al. (2008) at $43 \mathrm{GHz}(\lambda=7 \mathrm{~mm})$ with a high cadence in time. The first set of pilot observations indicated that a fast moving component could indeed be traced. This led to a program that produced a batch of eleven observations between 27 January 2007 and 26 August 2007, with an average time interval of 21 days, which allowed detection of apparent speeds as high as 3-4c. The result is a set of excellentquality, high-resolution VLBI maps with nearly homogeneous image rms noise. A more complete presentation of the 2007 data used here along with more densely sampled, but lower quality, data from 2008 plus roughly annual observations between 1990 and 2016 will be made separately (Walker et al., in prep.)

In the images of M87 obtained from the VLBA movie project, nearly the entire jet is well resolved, with up to 3-4 beamwidths across the flow. The limb-brightened morphology

\footnotetext{
1 Very Long Baseline Array of the National Radio Astronomy Observatory, USA.
}

is striking, especially if one looks at the stacked image that comprises the eleven observations into a single, high-sensitivity map. Determination of the velocity of the flow is however difficult from these data. The standard procedure for making proper motion measurements relies on modeling the source with a set of Gaussian components and cross-identifying those components between epochs. In this case, the complexity of the transversely resolved structure of the jet precludes application of this approach. Visual inspection was done in an attempt to identify apparently related features and a speed of about $2 c$ was consistently found (Walker et al. 2008). This method obviously lacks objectivity and robustness, and it is difficult to use it for obtaining a complete velocity field map of the jet.

A robust analysis of the M 87 VLBA movie dataset is required in order to make a quantitative assessment of the jet morphology and kinematics. Such an assessment can be made using the wavelet-based image segmentation and evaluation (WISE) method (Mertens \& Lobanov 2015) and the stacked cross-correlation algorithm (Mertens \& Lobanov 2016) developed for determining two-dimensional velocity fields. In the following a complete WISE analysis of the internal structure and dynamics of the jet in M87 at sub-parsec scale is presented. In Sect. 2, the WISE method is used to determine the twodimensional velocity field, and the collimation profile of the jet from the $43 \mathrm{GHz}$ VLBA observations of Walker et al. (2008). The complementary WISE analysis of the $15 \mathrm{GHz}$ VLBA observations from the MOJAVE project is presented in Sect. 3. The physical properties of the jet are discussed in Sect. 4. In Sect. 4.1, we present a physical model for the flow stratification observed in M 87. In Sect. 4.2, the viewing angle is derived from the velocity observed on the counter-jet side. In Sect. 4.3, evidence for jet rotation is discussed, as inferred from the differential velocity between the northern and southern limbs of the jet. Based on these findings, the acceleration and collimation of the jet are discussed in Sect. 4.4 in the framework of MHD jet acceleration. The physical nature of the jet spine is discussed in Sect. 4.5, and a plausible jet launching mechanism is proposed in Sect. 4.6.

Throughout the paper, a cylindrical coordinate system $(z, r$, $\phi$ ) is adopted for the jet description. In this coordinate system, the coordinates $z$ and $r$ correspond to axial and radial directions in the jet. We set the jet axis $(z)$ at a position angle of $20^{\circ}$ with respect to the RA axis, and we denote the projected axis as $z_{\text {obs }}$. The black hole mass estimates reported in the literature for M 87 are typically in the range of $\sim 3-7$ billion solar masses (cf. Walsh et al. 2013, and references therein). We adopt the value of $M_{\mathrm{BH}}=6.1 \times 10^{9} M_{\odot}$ in this paper.

\section{WISE analysis of $7 \mathrm{~mm}$ VLBA observations}

We applied WISE analysis (Mertens \& Lobanov 2015) to $11 \mathrm{im}-$ ages of the jet in M 87 obtained from $43 \mathrm{GHz}$ VLBA observations made between 27 January 2007 and 26 August 2007, with an average time interval of 21 days between the successive epochs and a common restoring beam of $0.43 \times 0.21$ mas in PA $-16^{\circ}$. We refer to Walker et al. (2008; in prep.) for a complete description of the data reduction. At the cadence of the $43 \mathrm{GHz}$ VLBA measurements, a displacement comparable to one fifth of the beam corresponds to a minimum detectable proper motion of $0.74 \mathrm{mas} / \mathrm{yr}$, or $0.19 c$. The WISE algorithm has been shown to provide robust detections of up to $\approx 4 F W H M$ between pairs of successive epochs (Mertens \& Lobanov 2015), which translates into a maximum apparent speed of $\sim 5 c$ that can be detected from the VLBA images of M 87. Hence, the WISE analysis should be able to detect essentially the full range of speeds reported in the compact jet of M 87 . 

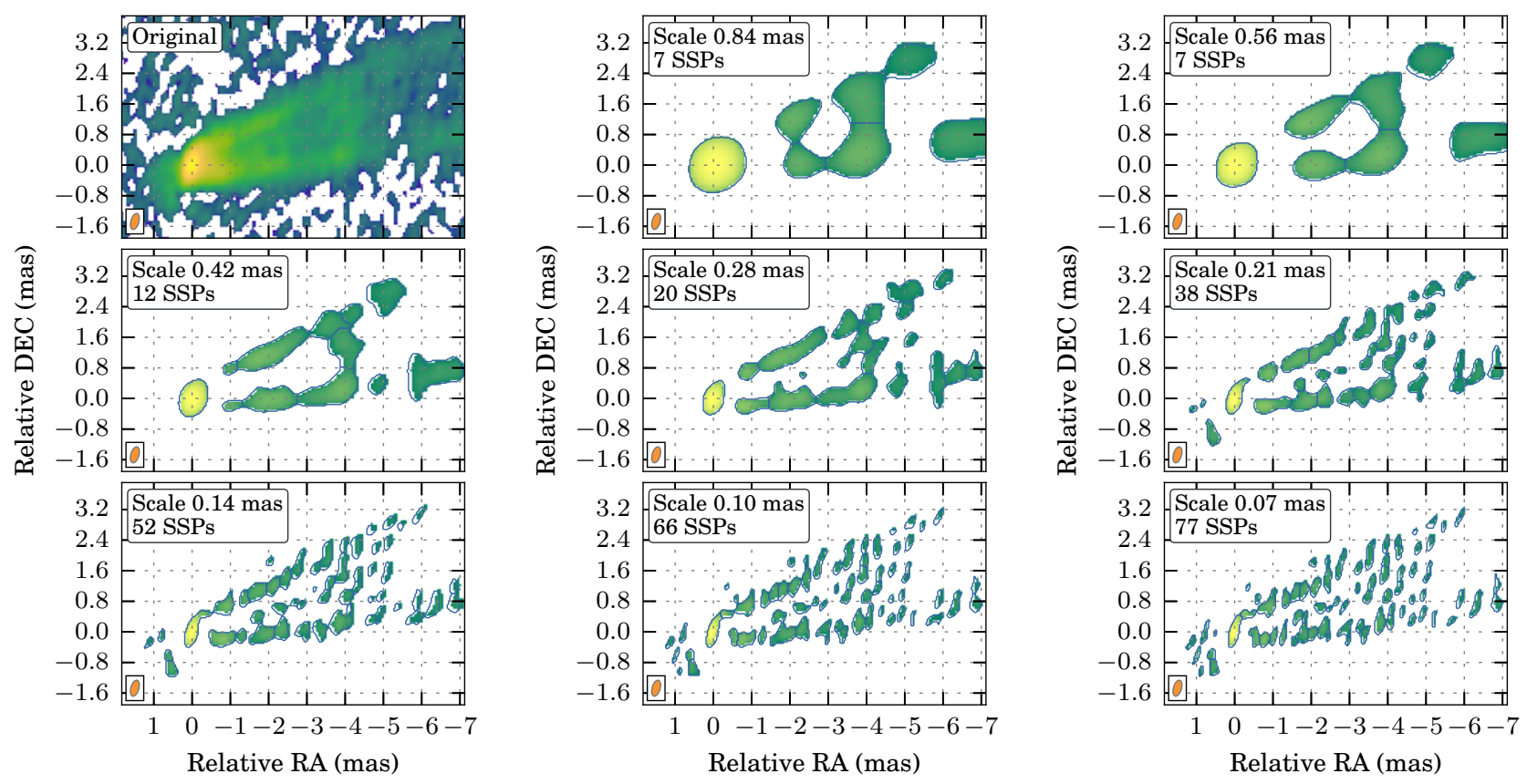

Fig. 1. $43 \mathrm{GHz}$ VLBA image of the jet in M 87 at the epoch 2007/02/17 (top left panel) and its WISE decomposition on SWD scales 1-4 (0.07, $0.14,0.28,0.56 \mathrm{mas})$ and IWD scales $(0.105,0.21,0.42,0.84 \mathrm{mas})$.

\subsection{Velocity field analysis}

Analysis of the M87 images is performed as described in Mertens \& Lobanov (2015). Each map is decomposed and segmented using the segmented wavelet decomposition (SWD) method, which provides a description of the two-dimensional jet structure based on a set of significant structural patterns (SSP). These features are then cross-identified in pairs of adjacent epochs using the multiscale cross-correlation (MCC) method. This procedure yields spatial displacements and proper motions for each of the SSPs identified.

To ensure robust detectability of displacements larger than one beamwidth (corresponding to apparent speeds of $z 2 c$ ), we applied the SWD on four spatial scales (ranged as powers of two) of $0.07,0.14,0.28$, and 0.56 mas and amended them with the intermediate wavelet decomposition (IWD) performed on scales of $0.105,0.21,0.42$, and 0.84 mas. The IWD implementation, described in Appendix A, is made to improve the efficiency of the MCC algorithm for recovering structural changes in optically thin, stratified flows. With these settings, maximum displacements of about 0.84 mas (corresponding to proper motion of $\sim 18 \mathrm{mas} / \mathrm{yr}$ and $\beta_{\mathrm{app}} \sim 4.5 \mathrm{c}$ ) can be robustly detected in each successive pair of images even for overlapping optically thin regions with different apparent speeds. The noise was estimated by computing $\sigma_{j}$ at each wavelet scale, as described in Mertens \& Lobanov (2015) and a $3 \sigma_{j}$ thresholding was subsequently applied at each scale. The typical $\mathrm{S} / \mathrm{N}$ found in different parts of the jet and for different Scales is summarized in Table 1.

Figure 1 shows an example of the SWD/IWD decomposition of the VLBA image of M 87 from the epoch 2007/02/17. The internal structure of the flow is well sampled at Scales 1 and 2 of the SWD, while the SWD Scales 3 and 4 are essential for providing an effective reference frameset for the successful determination of the SSP displacement made using the MCC algorithm.
Table 1. Median $S / N$ of SSPs detected in different parts of the jet and for different scales.

\begin{tabular}{cccc}
\hline \hline Region & Scale 1 & Scale 2 & Scale 3 \\
\hline Limbs, $z_{\text {obs }} \leq 4$ mas & 17.2 & 20.6 & 61.9 \\
Limbs, $z_{\text {obs }}>4$ mas & 6.4 & 7.6 & 11.2 \\
Center, $z_{\text {obs }} \leq 4$ mas & 7.3 & 9.2 & - \\
Center, $z_{\text {obs }}>4$ mas & 6.8 & 7.4 & 5.0 \\
\hline
\end{tabular}

The MCC algorithm has been applied to each consecutive pair of the jet images. Based on the previously reported measurements made at these distances in the jet (Reid et al. 1989; Biretta et al. 1995; Dodson et al. 2006; Kovalev et al. 2007), one can expect the jet to manifest a mixture of large and small displacements. To ensure robust detectability of the entire range of potential displacements, we use a tolerance factor of 1.5 and a correlation threshold of 0.6 in our MCC analysis. Finally, velocity constraints are set according to the expected largest displacement ranges: on the jet side, the longitudinal velocities are allowed to range between -2 mas/yr and +18 mas/yr, transversally they are allowed to range between -5 mas/yr and +5 mas/yr. On the counter-jet side, velocities ranging between -2 mas/yr and $2 \mathrm{mas} / \mathrm{yr}$ longitudinally and transversally are allowed. For the analysis of the fine two-dimensional velocity structure of the jet, only the displacements determined at the lowest SWD scale (Scale 1: 0.07 mas) are considered. The displacements determined at coarser scales were used in the MCC and overall are consistent with the ones obtained at the 0.07 mas scale.

The SWD yields a total of 68 SSPs that are detected on average in each single image of the jet. At the smallest spatial scale of the SWD, SSP matching is successfully made for $51 \%$ of the SSPs, resulting in detection of a total of 391 displacement vectors. The normalized cross correlation between matching sets of SSPs is larger than 0.8 , on average. The errors of the displacements and velocity vectors are obtained from the uncertainties of the SSP positions which are computed using the 


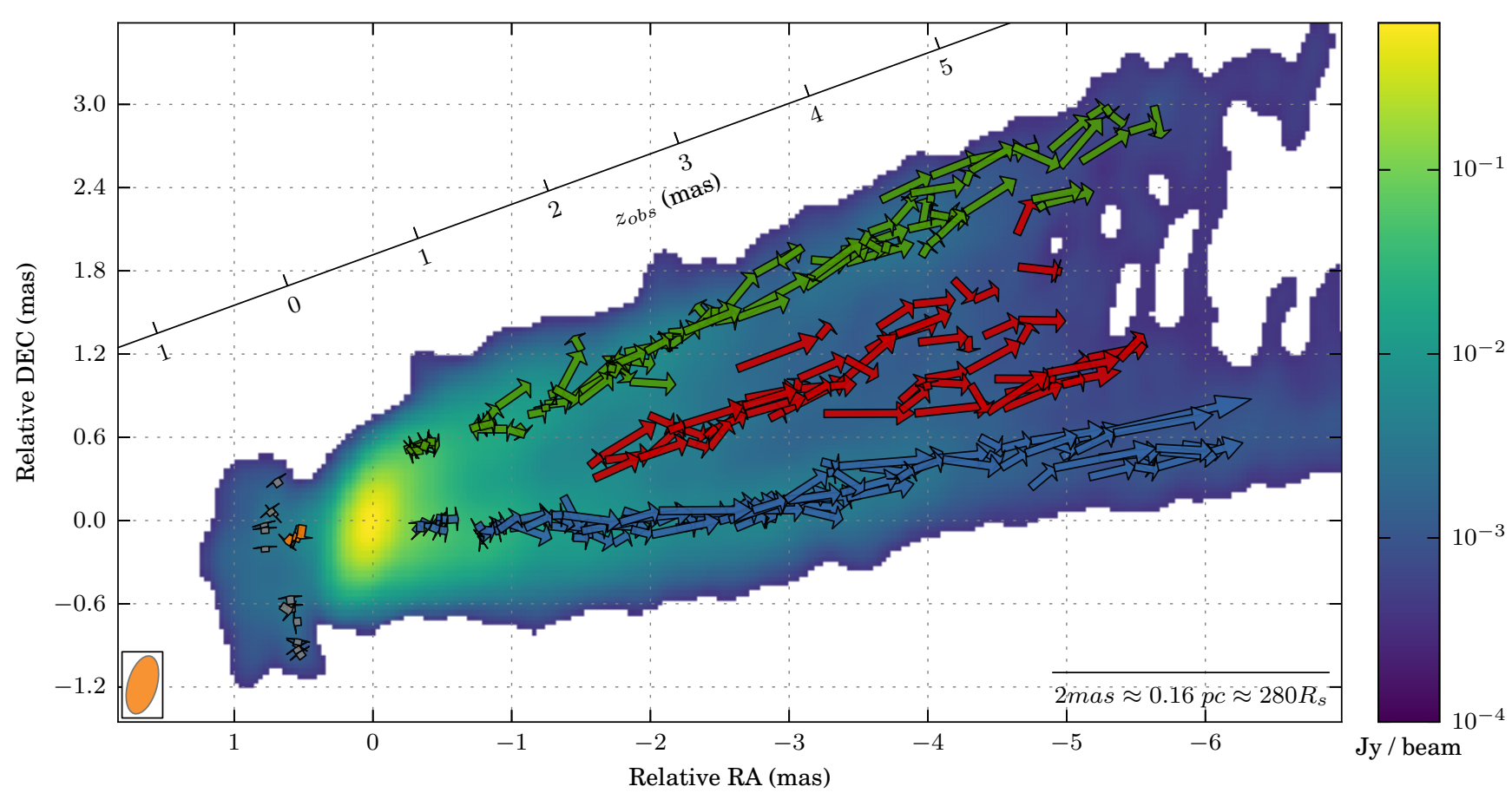

Fig. 2. Observed displacements of the significant structural patterns detected in the jet of M 87 . Three main regions can be identified in the jet, as revealed by consistency between the individual displacements in the southern (blue) and northern (green) limbs and in the central "stream" (red) of the flow. The components found in the inner part of the counter-jet (orange), may reflect motions in a slow, weakly deboosted wind on the counter-jet side. In the outer part of the counter-jet (gray), a slower transversal motion is evident, with a tendency toward the northward direction. The displacements are overplotted on a stacked-image of the jet clipped at a $5 \sigma$ noise $\mathrm{rms}$ level $(0.5 \mathrm{mJy} / \mathrm{beam})$.

stacked cross-correlation of the SSP (Mertens \& Lobanov 2016), which yields:

$\sigma_{x}=\frac{b_{x}}{\sqrt{2} S / N}, \sigma_{y}=\frac{b_{y}}{\sqrt{2} S / N}$,

where $b_{x}$ and $b_{y}$ describe the beam size along the $x$ and $y$ coordinates, respectively, and $\mathrm{S} / \mathrm{N}$ is the signal-to-noise ratio of the SSP detection.

\subsection{SSP displacements and jet kinematics}

The SSP displacements obtained using this approach are shown in Fig. 2, and the transverse and longitudinal components of the corresponding velocities are plotted in Fig. 3.

Figures 2, 3 indicate that the jet exhibits three kinematically distinct regions of organized stream-like features with predominantly longitudinal displacement vectors. These features will hereafter be referred to as the northern and the southern limbs and the central stream (we prefer not to use the term "spine" for this feature as it is not clear whether it is indeed related to the physical relativistic spine of the stratified outflow in M 87).

The kinematics is more complex in the central stream region which manifests both outward motions and substantial transverse displacements. The displacements can be reliably detected in the central stream at $z_{\mathrm{obs}} \gtrsim 1.75$ mas, when the width of the jet exceeds three beam sizes.

The top panel of Fig. 3 shows the presence of both slow (subluminal) and fast (superluminal) speeds in the central stream and each of the limbs. This spread of apparent speeds might indicate jet stratification. In each of the three regions, we also detect several outwards motions which are most of the time compatible with no motion (taking into account position uncertainty) but might also be related to instability pattern motion.

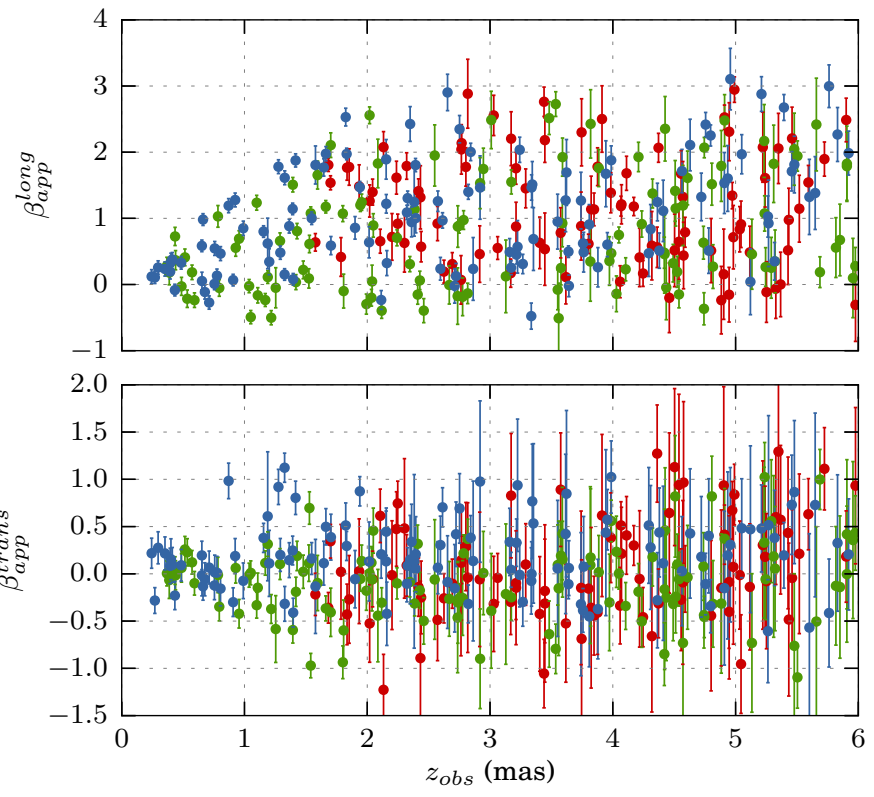

Fig. 3. Longitudinal (top) and transverse (bottom) velocities of significant structural patterns identified in the M 87 jet. Colors indicate respective jet regions in which given SSPs are located (following the color coding introduced Fig. 2): green and blue mark the SSPs from the northern and southern limb, respectively, and red marks the SSPs from the central stream.

An apparent acceleration can be seen in both limbs over the innermost 2 mas of the jet. At a distance $z_{\text {obs }} \sim 0.25$ mas, the maximum apparent velocity is $\beta_{\text {app }} \sim 0.2 c$, which then linearly increases up to $\beta_{\text {app }} \sim 2.5 \mathrm{c}$ at $z_{\text {obs }} \sim 2$ mas. Between $z_{\text {obs }} \sim 2$ mas and $z_{\text {obs }} \sim 6$ mas, we measure either a slow acceleration or 
no acceleration at all. In this region, a maximum velocity of $\beta_{\text {app }} \sim 3 c$ is found at $z_{\text {obs }} \sim 5$ mas. We note that because this acceleration is observed at the limbs of the jet, the measured apparent velocities are only marginally affected by the jet opening angle (see Eq. (6)), and a fast decrease in opening angle between 0 and 2 mas would not be able to explain the observed increase in apparent velocity as this decrease would strongly affect only the velocities measured in the central stream, which is not detected at distances smaller than $\approx 2$ mas.

We also observe an oscillation of the speed measured along the jet, with minima in the jet speed found at $z_{\mathrm{obs}} \sim 1.2 \mathrm{mas}$, 2.25 mas and 4.25 mas.

On the counter-jet side, there is only one SSP component that shows consistently outward motion over five subsequent epochs (orange arrows in Fig. 2). At this distance from the core, the counter-jet also appears limb-brightened, and the apparent position of this feature is consistent with it being located in the northern limb. We can use it's speed and brightness relative to the main jet to obtain estimates of the jet angle to the line of sight and intrinsic speed.

Several other features in the outer part of the counter-jet exhibit slower motions tending toward the northward direction, which may be viewed as a hint for the clockwise rotation, as viewed by the observer, of the emitting material. The robustness of detection of the displacements of these features and potential physical interpretation of these displacements should be a matter of further, more detailed investigation.

In order to assess the robustness of the SSP and displacement identification, we perform a bootstrapping analysis by randomly shuffling the individual images and carrying out the WISE analysis on a number of such random sets. We found that on average only $35 \%$ of the SSPs can be matched in the pairs of shuffled images and only $13 \%$ of those can be traced over five or more epochs, compared to $24 \%$ for the non-shuffled set of images. The bootstrapping datasets also do not produce regular velocity patterns and do not show the correlation between $z_{\text {obs }}$ and $\beta_{\text {long }}$ observed in the original sequence of images. This demonstrates that the solution found by WISE does not result from a spurious correlation.

\subsection{Flow stratification analysis}

The large variations of velocity measured along and across the jet indicate the complex physical nature of the flow, with both acceleration and stratification of the flow likely to play important roles. To investigate the potential effect of flow stratification on the observed velocity field we combine the WISE velocity field analysis with the stacked cross correlation (SCC) analysis (Mertens \& Lobanov 2016). The SCC method combines the cross-correlation results for all SSPs identified at different scales of the SWD in all different pairs of epochs. This procedure yields a two-dimensional distribution of the cumulative correlation coefficients, with peaks corresponding to different velocity components. The significance and uncertainty of each of these peaks is evaluated using Monte Carlo simulations.

For the present analysis, the SSPs detected at the three lowest SWD scales are used. The jet is divided in two different regions (region "A" at 0.5-1.0 mas distance from the core and region "B" at 1-4 mas distance from the core), and the SCC analysis is performed independently for each of these regions. The extent of these two regions is constrained by the requirement for the detection of statistically significant velocity components in the SCC analysis of both the limbs and the central stream of the flow.
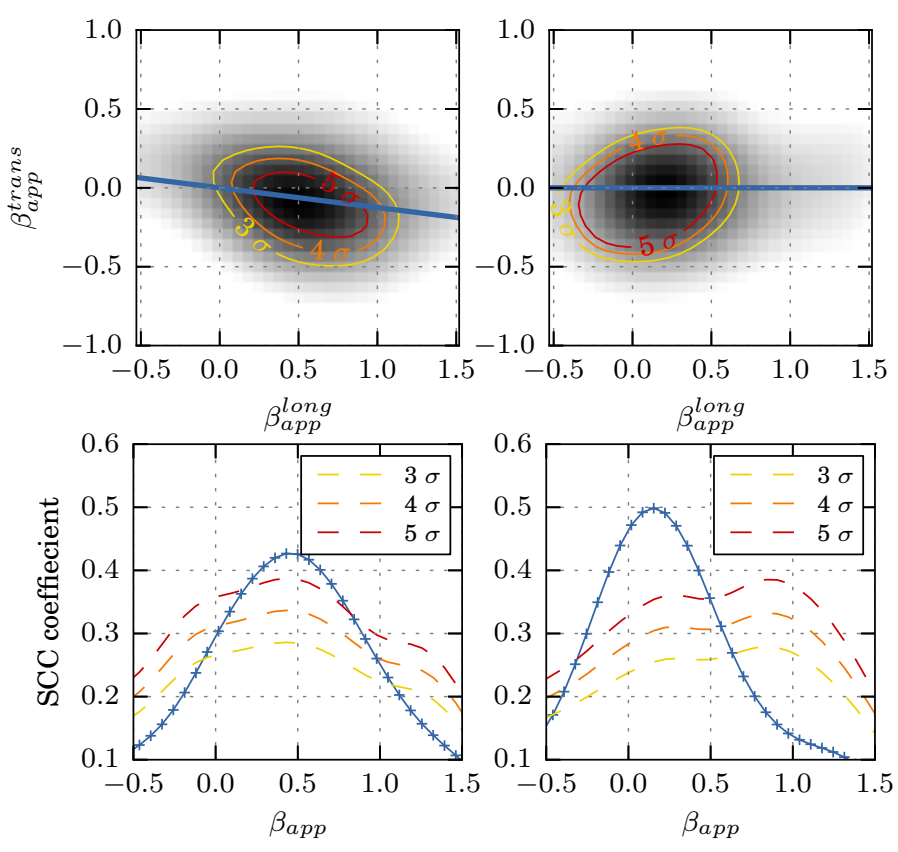

Fig. 4. Results of the stacked cross correlation analysis of the northern (top left panel) and southern limbs (top right panel) in Region " $\mathrm{A}$ " of the jet at distances between 0.5 and 1 mas from the core. The velocity cross correlation map is shown at the top in gray scale. Contours indicate the statistical significance of the correlation. A slice along the blue line is shown in the bottom panels. A single velocity component with more than $5 \sigma$ significance is found in both limbs. The parameters for this velocity component are listed in Table 2.

\subsubsection{Region "A"}

In the northern part of the jet, the SCC is computed from a total of 42 SSPs. The resulting velocity cross correlation maps and the corresponding significance estimates are plotted in Fig. 4. For the northern limb of region "A", the SCC results imply a single velocity component, with an apparent speed of $\beta_{\text {app }}^{\text {north }}=0.48 \pm$ $0.06 c$ and a significance of $5.9 \sigma$. In the southern limb, the SCC has been applied to a total of 47 SSPs, also revealing a single velocity component with $\beta_{\text {app }}^{\text {south }}=0.21 \pm 0.04 c$ and a significance of $8.7 \sigma$. The results are further summarized in Table 2 .

The SCC analysis did not unveil any stratification close to the core. This result confirms however the speeds measured in Sect. 2.1, further strengthening the conclusion that subluminal speeds are generally found in this region. If any stratification would be present, the respective velocity difference must have been too small so that it would remain undetected with the SCC. In this case, the apparent velocity measured by this method can be interpreted as a weighted mean velocity of the different jet layers along a given line of sight. This may also explain the difference between the speeds measured in the northern and southern limb (an alternative explanation may involve rotation of the flow, which will be examined later).

\subsubsection{Region "B"}

For region "B" (1-4 mas from the core), the SCC analysis was performed independently for each of the limbs and for the central stream of the jet, using 245, 267, and 217 SSPs, respectively. The resulting cross-correlation maps are plotted in Fig. 5. The measured correlations reveal the presence of two distinct velocity components, which suggest a strong stratification of the flow with a slow, mildly relativistic speed $\left(\beta_{\text {app }} \sim 0.35 c\right)$ and a faster, 

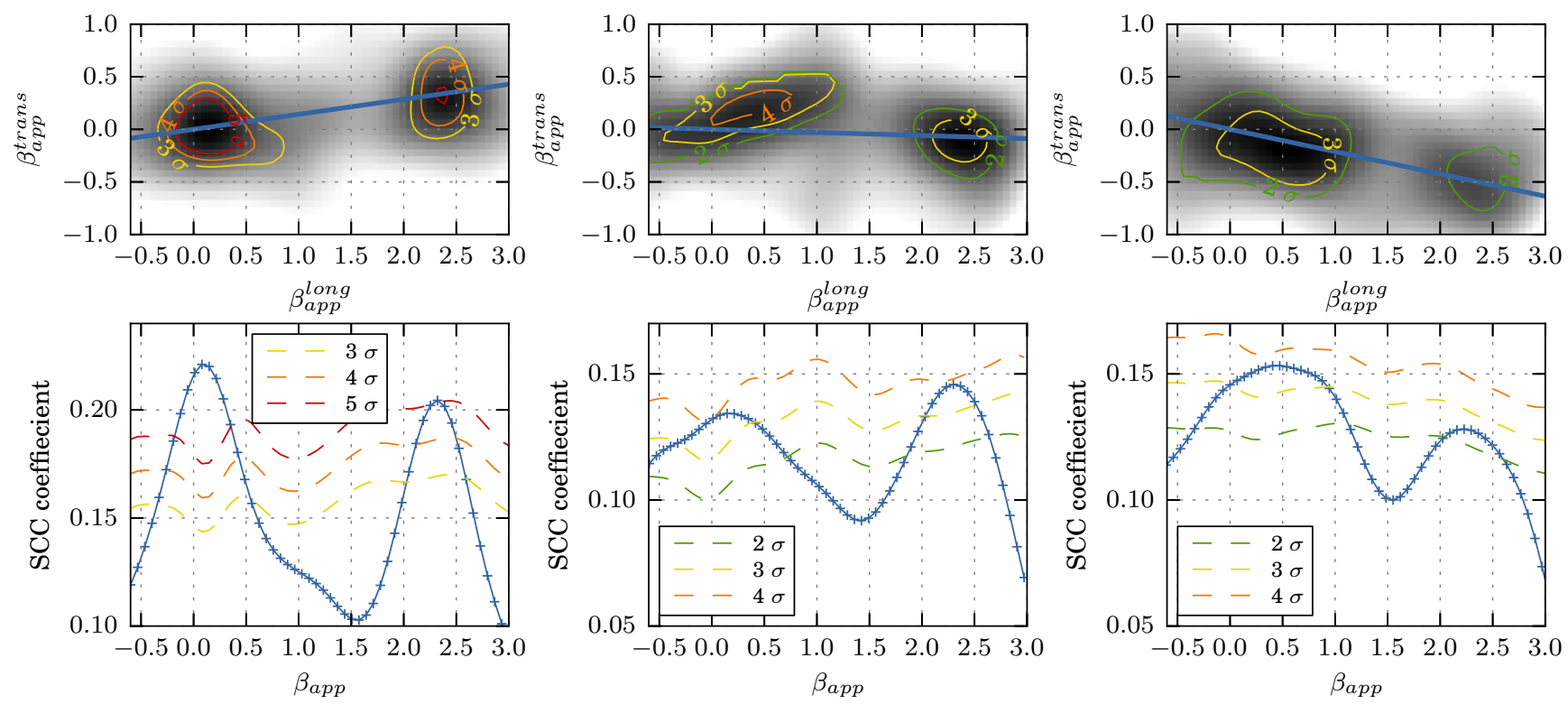

Fig. 5. Results of the stacked cross correlation analysis of the northern limb (right panel), central stream (middle panel), and southern limb (left panel) in Region " $\mathrm{B}$ " of the jet, at a distance between 1 and 4 mas from the core. The cross correlation map is shown at the top in gray scale along with contours of the significance. A slice along the blue line is shown at the bottom. Two main velocity components are consistently found in the three regions of the jet. The derived parameters for the velocity components are listed in Table 3.

Table 2. Velocity components identified from stacked cross correlation analysis of the northern and southern limbs in Region "A", at distances between 0.5 and 1 mas from the core.

\begin{tabular}{ccccc}
\hline \hline Region A & $\beta_{\text {app }}^{\text {trans }}$ & $\beta_{\text {app }}^{\text {long }}$ & $\beta_{\text {app }}$ & $\sigma$ \\
\hline North & $-0.10 \pm 0.03$ & $0.47 \pm 0.05$ & $0.48 \pm 0.06$ & 5.9 \\
South & $-0.02 \pm 0.03$ & $0.21 \pm 0.03$ & $0.21 \pm 0.04$ & 8.7 \\
\hline
\end{tabular}

Notes. $\sigma$-statistical significance of the SCC peak.

Table 3. Velocity components identified using the stacked cross correlation analysis in Region "B", at 1-4 mas distance from the core.

\begin{tabular}{lcccc}
\hline \hline Region B & $\beta_{\text {app }}^{\text {trans }}$ & $\beta_{\text {app }}^{\text {long }}$ & $\beta_{\text {app }}$ & $\sigma$ \\
\hline \multicolumn{2}{l}{ Fast velocity component } & & & \\
North & $0.27 \pm 0.04$ & $2.40 \pm 0.03$ & $2.41 \pm 0.05$ & 5.1 \\
Center & $-0.1 \pm 0.1$ & $2.32 \pm 0.17$ & $2.32 \pm 0.20$ & 4.0 \\
South & $-0.41 \pm 0.07$ & $2.16 \pm 0.14$ & $2.20 \pm 0.15$ & 2.5 \\
Slow velocity component & & & \\
North & $0.01 \pm 0.03$ & $0.17 \pm 0.04$ & $0.17 \pm 0.06$ & 8.0 \\
Center & $0.13 \pm 0.12$ & $0.34 \pm 0.30$ & $0.37 \pm 0.32$ & 4.4 \\
South & $-0.14 \pm 0.07$ & $0.47 \pm 0.22$ & $0.49 \pm 0.24$ & 3.9 \\
\hline
\end{tabular}

Notes. $\sigma$-statistical significance of the SCC peak.

relativistic speed $\left(\beta_{\text {app }} \sim 2.3 c\right)$. The velocity parameters derived for these components are summarized in Table 3.

The statistical significance of most of the velocity components found in region " $\mathrm{B}$ " is better than $\approx 4 \sigma$. The overall consistency of the speeds found in the limbs and the central stream lends further support for stratification of the flow. A somewhat low $(2.5 \sigma)$ formal significance of the correlation is found for the fast velocity component in the southern limb of the jet. This may result from a broader range of measured displacement velocities registered there as compared to the northern limb.

\subsection{Jet collimation}

In the magnetically accelerated jet model, the conversion from Poynting flux into kinetic energy depends on the configuration of the magnetic field lines (Begelman \& Li 1994; Lyubarsky 2010). The jet collimation profile is therefore an important indicator of the jet acceleration mechanism. The segmented wavelet decomposition (SWD) method introduced in Mertens \& Lobanov (2015) provides a reliable way to analyze the evolution of the jet width (jet radius) with distance from the core.

To determine the jet collimation profile, three stacked-epoch wavelet scale (SWS) images of M 87 are prepared. Each of these images comprises all SSPs detected at a given scale $j=1,2,3$ at all 11 epochs. Near to the jet base, the jet limb is well described by the SWS at the smallest wavelet scale. As the jet expands, the width of each of its limbs is best described by progressively larger SWS scales. Scale 1 (0.07 mas) of the SWS is used between $z_{\text {obs }}=0.4$ mas and $z_{\text {obs }}=0.8$ mas, scale $2(0.14$ mas $)$ is then used up to $z_{\mathrm{obs}}=1.6 \mathrm{mas}$, and finally scale $3(0.21 \mathrm{mas})$ is selected at larger core separations.

The jet radius is estimated by taking transverse profiles of the stacked-epoch SWS images of the jet at regular intervals $(\delta z=$ $1 \mathrm{px}=0.035$ mas) and measuring the distance between the two limbs of the jet. A Gaussian profile is fit to each of the limbs to further improve this calculation. It is particularly difficult to obtain a robust uncertainty estimate for these measurements. Our conservative approach is to assume that the position error of limb determination is $1 / 4$ of the beam $(0.07$ mas $)$ at the core of the jet, and linearly increases to reach $1 / 2$ of the beam at the last profile, located at 6.6 mas from the core. The result is plotted in Fig. 6. The instantaneous apparent jet opening angle, $\Theta_{\text {app }}$, is computed from the measured jet radii using the relation $\Theta_{\text {app }}=$ $\arctan \left(\mathrm{d} r / \mathrm{d} z_{\text {obs }}\right)$. The local curvature $\mathrm{d} r / \mathrm{d} z_{\text {obs }}$ is approximated from a linear fit of the jet radius 0.2 mas before and after each point of the calculation.

The jet collimation profile is well described by a power law $r \propto z^{k}$ with $k=0.60 \pm 0.02$ (reduced $\chi^{2}=0.55$ ). This result is consistent with previous measurements made by 

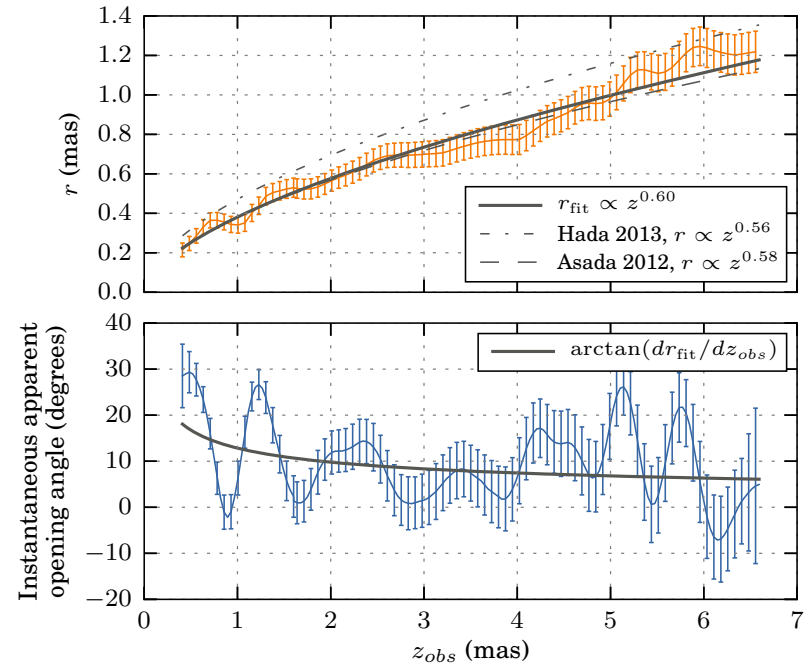

Fig. 6. Evolution of the radius (top panel) and instantaneous apparent opening angle (bottom panel) of the jet in M 87 with distance from the core. A power law fit to the jet radius is consistent with results obtained by Asada \& Nakamura (2012; dashed line) and Hada et al. (2013; dashed dotted line). Evolution of the instantaneous opening angle corresponding to the power law fit obtained for the jet radius is shown by the solid gray line in the bottom panel.

Asada \& Nakamura $(2012 ; k=0.58 \pm 0.02)$ and Hada et al. (2013; $k=0.56 \pm 0.03)$. As seen from Fig. 6, expansion of the jet reveals an oscillatory pattern, most likely reflecting repeated over-collimation and over-expansion of the flow. The over-collimation is visible at $z_{\mathrm{obs}} \sim 1,1.9$ and 4 mas, and the over-expansion manifests itself at $z_{\mathrm{obs}} \sim 0.75,1.4$ and 2.6 mas, suggesting that the spatial period of these oscillations increases with the distance as $\sim z_{\text {obs }}$. Oscillations of the jet width seen at distances $\gtrsim 5$ mas have a shorter period of $\approx 0.5$ mas.

It is interesting to note that oscillations of the jet width correlate with the changes in the apparent jet speed reported in Sect. 2.2. The locations of maximum over-collimation of the jet correspond well to the observed minima in the apparent speed of the jet. If these oscillations reflect the hydrodynamics of the flow, the pressure ratio between the jet and the external medium might be close to unity (Daly \& Marscher 1988). Presence of oscillating patterns in an expanding flow is also expected for an expanding magnetized jet (Lyubarsky 2009; Komissarov et al. 2015). Alternatively, these oscillations might reflect the evolution of the pinch mode or the elliptical surface mode of K-H instability (Hardee 2000; Lobanov \& Zensus 2001).

\section{WISE analysis of $2 \mathrm{~cm}$ VLBA observations}

Between 1995 and 2010, the jet in M 87 was regularly observed as part of the MOJAVE project (Lister et al. 2009, 2013). The MOJAVE database contains 30 images of this jet, providing, on average, one observation every seven months. With this cadence, WISE can reliably detect jet speeds of up to $\beta_{\text {app }} \sim 2 c$, assuming a maximum detectable displacement of four times the beam size. In an analysis of this data using the Gaussian model-fitting technique, Kovalev et al. (2007) reported only a slow, subluminal motion with a maximum speed $\beta_{\text {app }} \sim 0.05 \mathrm{c}$. Finding this result to contradict several physical aspects of the jet, including the counter-jet flux density ratio, Kovalev et al. (2007) suggested that the measured subluminal speed might represent pattern motions of either shocks or MHD instabilities. Using the WISE analysis, we can attempt to detect a faster speed from

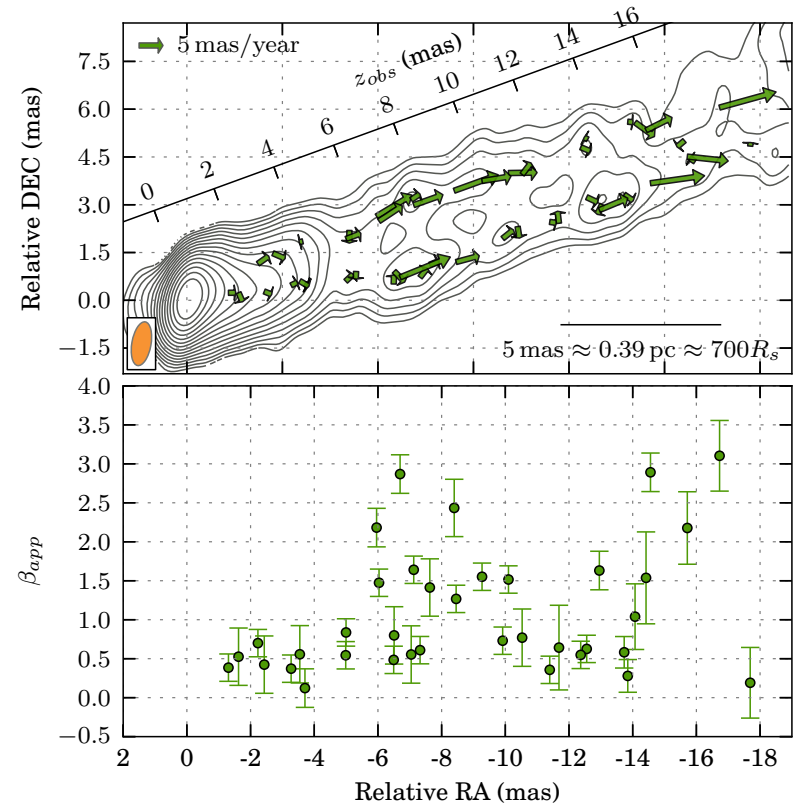

Fig. 7. Two-dimensional velocity field traced by the structural patterns detected using the WISE analysis of four epochs of $15 \mathrm{GHz}$ VLBA observations of M 87 made between 2000/01/07 and 2000/06/27 (upper panel). The tracks are overplotted on a stacked-epoch image of the jet. The corresponding apparent velocities are plotted in the bottom panel.

the MOJAVE data. The application of the SWD made for this purpose is performed using four scales, ranging from 0.4 mas (scale 1) to 3.2 mas (scale 4).

The interval between two epochs is generally too long to warrant detection of the fast velocity $\beta_{\mathrm{app}} \sim 2.5 c$ that has been measured in the $43 \mathrm{GHz}$ VLBA data. We concentrate therefore on the pair of observations separated by the shortest time interval. The complete MOJAVE database has two epochs (2000/04/07 and 2000/06/27) that matched this criteria, and we complement them with two full track $15 \mathrm{GHz}$ VLBA observations obtained at epochs 2000/01/22 and 2000/05/08 (Kovalev et al. 2007).

The result of applying the WISE algorithm to these four VLBA images at $15 \mathrm{GHz}$ is plotted in Fig. 7. We detected fast relativistic speeds of $\beta_{\text {app }} \sim 2-3 c$ for six different SSPs in both the northern and southern limbs of the jet at $z_{\text {obs }} \sim 6.5$ mas and $z_{\text {obs }} \sim 16$ mas. In addition to this, eight SSPs exhibit apparent superluminal speeds of $\beta_{\text {app }} \sim 1.5 c$, and subluminal speeds $\left(\beta_{\text {app }} \sim 0.5 c\right)$.

The displacements traced in the $15 \mathrm{GHz}$ VLBA images do not provide enough material for tracing the velocity field in the jet. However, one can see that full-tracks VLBA observations at $15 \mathrm{GHz}$ made at $\sim 2$ month intervals should be sufficient to obtain a reliable velocity field.

\section{Discussion}

\subsection{Jet internal structure}

The stacked cross correlation analysis discussed in Sect. 2.3 reveals a clear stratification of the flow, with a slow, subluminal component and a faster relativistic component detected in all three streams inside the jet. Both the slow and the fast velocity components exhibit similar speeds in each of the streams, which suggests that each of them reflects plasma motions occurring at similar radial separations (similar jet layers) in a transversally 


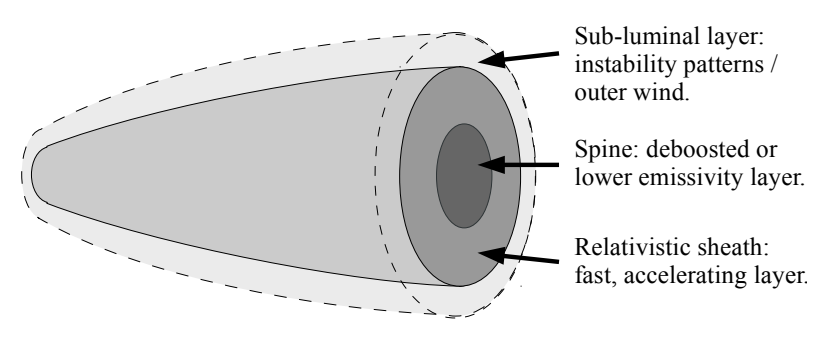

Fig. 8. Schematic illustration of the internal structure of the M 87 jet adopted in the analysis presented in this paper. The superluminal kinematic feature in the jet is associated with the sheath and the subluminal feature is related to a slower, outer layer of the flow. The flow is also expected to feature a fast inner spine, which however cannot be detected in the present data either because of its lower emissivity compared to the emissivity of the sheath layer or because its speed is too high to be detected with the present data.

stratified flow. Since the fast velocity component is present in all three streams, it can be associated with the sheath, rather than the spine, of the jet. A faster spine is either not present in the flow (at least at the linear scales studied here), or it is deboosted and appears dimmer than the slower moving material of the sheath (in this case, VLBI observations at time intervals of smaller than 10 days are required to successfully disentangle this faster component from the two velocity components identified in this paper). In further analysis, we assume the latter possibility, which corresponds to the schematic view of the jet shown in Fig. 8 .

The observed jet stratification, with a fast, accelerating sheath and a subluminal layer can be interpreted in several different ways. It may reflect a "two-fluid" jet (Sol et al. 1989) comprising a relativistic beam (or spine) originating either from the magnetosphere of the central engine, or from the inner part of the accretion disk, and a non-relativistic wind originating from outer parts of the accretion disk (Tsinganos \& Bogovalov 2002). The viability of this scenario has been tested in RMHD simulations of AGN jets (e.g., Komissarov et al. 2007) which demonstrated that magnetic field lines originating from different parts of the accretion disk would result in substantial transverse stratification of the flow.

Alternatively, the fast sheath component may reflect the true speed of the flow (without substantial transverse stratification), while the slower one would then be interpreted as a pattern speed associated with current driven (CD) or Kelvin-Helmholtz (K-H) instability. In general, K-H instability pattern speed is expected to be considerably slower than the flow speed (Hardee 2000). It was also suggested that the subluminal apparent speeds of $\sim 0.5 c$ detected in the jet in M 87 at kiloparsec scale indicate a pattern speed from K-H instability (Lobanov et al. 2003).

\subsection{Counter-jet and jet viewing angle}

The presence of a counter-jet in M87 jet has been suggested based on the structure detected in $15 \mathrm{GHz}$ VLBA maps (Kovalev et al. 2007). A similar structural detail resembling a counter-jet is also observed in the $43 \mathrm{GHz}$ images of M 87 (Ly et al. 2007; Walker et al. 2008). The WISE analysis performed on the $43 \mathrm{GHz}$ VLBA data has revealed several SSPs located in the counter-jet structure and moving outward with speeds similar to the subluminal speed measured on the jet side at the same distances from the core. We will use this velocity measurement to constrain the viewing angle of the jet.

Relativistic Doppler beaming affects differently the flux density in the jet and the counter-jet. If the jet is seen at a viewing

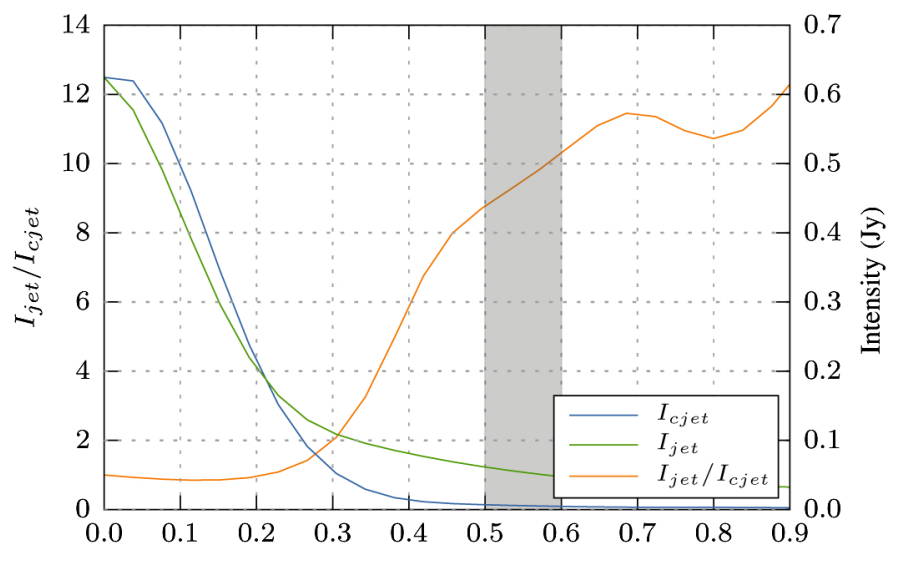

Fig. 9. Jet (green) and counter-jet (blue) longitudinal intensity profile and their corresponding ratio (orange). The gray shading indicates the span travelled by the SSP detected on the counter-jet side for which the speed has been robustly estimated. In order to exclude biasing by the bright core of the jet, only measurements at core separations larger than 0.4 mas $(\approx 1.4 F W H M$ of the restoring beam) are considered in the analysis of the jet/counter-jet ratio.

angle $\theta$, the observed intensity ratio between the jet and counterjet is given by:

$\mathcal{R}=\frac{I_{\mathrm{jet}}}{I_{\mathrm{cjet}}}=\left[\frac{\gamma_{\mathrm{cj}}\left(1+\beta_{\mathrm{cj}} \cos (\theta)\right)}{\gamma_{\mathrm{j}}\left(1-\beta_{\mathrm{j}} \cos (\theta)\right)}\right]^{2-\alpha}$,

with $\beta_{\mathrm{j}}, \beta_{\mathrm{cj}}$ are the intrinsic speeds of the plasma in the jet and counter-jet respectively, $\gamma_{\mathrm{j}}, \gamma_{\mathrm{cj}}$ are the corresponding Lorentz factors and $\alpha$ is the spectral index of the synchrotron radiation, $I_{v} \propto v^{\alpha}$. In a recent publication, $\alpha=-1$ was estimated for the radio emission on sub-parsec scales in the M 87 jet (Hovatta et al. 2014).

We measure the intensity ratio $\mathcal{R}$ by taking a longitudinal profile of emission brightness along the jet limbs. In order to reference properly the jet and counter-jet locations, special attention must be paid to the identification of the position of the core. Because of synchrotron self-absorption, the observed location of the jet core does not correspond exactly to the true base of the jet (Lobanov 1998). However, in the case of M 87, Hada et al. (2011) found that the central engine of M 87 and the radio core at $43 \mathrm{GHz}$ are separated by a projected distance of only $41 \pm 12 \mu \mathrm{as}$, which is small enough to consider, in our case, the core as the point of symmetry of the jet. The counter-jet component which yields a robust speed estimate is located in the northern limb of the counter jet. To preserve the symmetry, the jet to counterjet intensity ratio is measured between the northern limb of the counter jet and the southern limb of the jet. To obtain the profile, flux density is measured at every pixel ( 0.035 mas; $1 / 8$ of the beam size). The result is shown in Fig. 9. We note here again that both the intensity ratio and velocities are measured at the limbs of the jet, and therefore the effect of variable jet opening angle near the core would only be marginal and well below the statistical uncertainties of our measurements.

In order to avoid biasing by the bright core of the jet we exclude the inner 0.4 mas portion of the flow (corresponding to $\approx 1.4 \mathrm{FWHM}$ of the restoring beam). At separations between 0.4 mas and 0.8 mas, we observe a sharp increase of the jet to counter-jet intensity ratio from $\sim 7$ to $\sim 11$ which may result from acceleration of the flow. In this region, robust displacement detections have been made in three components on the jet side (with speeds ranging from $0.16 c$ to $0.24 c$ ) and in one feature on 


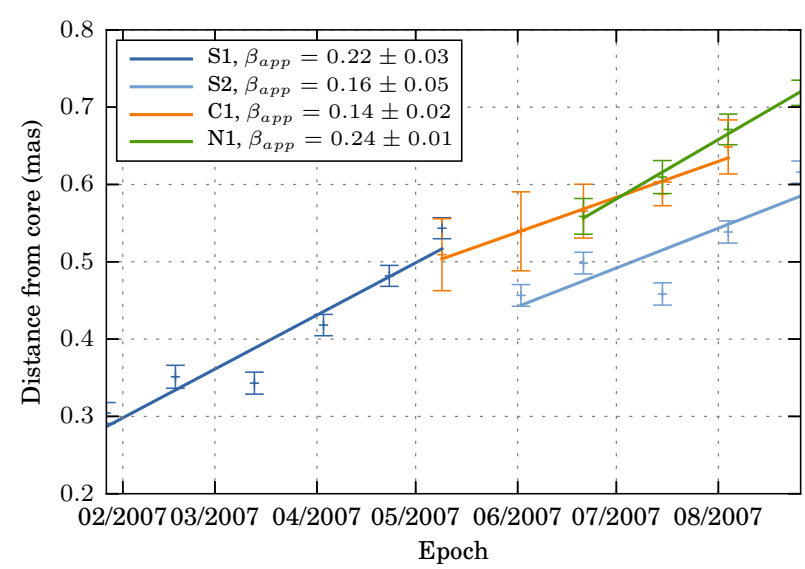

Fig. 10. Core separations of the SSPs that were tracked for at least four epochs over a 0.8 mas extent in both the jet and counter-jet direction. The jet components S1 (dark blue) and S2 (light blue) are detected in the southern limb of the jet, while the jet component N1 (green) is detected in the northern limb of the jet. On the counter jet side, one component, $\mathrm{C} 1$ (orange) is detected in the northern limb of the flow.

the counter-jet side with an apparent speed of $0.14 c$. These components are presented in Fig. 10.

The combination of the observed jet to counter-jet intensity ratio and the maximum and minimum measured apparent speeds provides conservative constraints on the viewing angle of the jet. This is illustrated in Fig. 11 which shows the ranges of the viewing angle and the intrinsic speed that can reconcile both the apparent speed and the measured jet to counter-jet intensity ratio:

$13^{\circ} \leq \theta \leq 27^{\circ}, 0.32 \leq \beta \leq 0.4$.

This estimate assumes that both the jet and the counter-jet have the same intrinsic speed and brightness. In a stratified flow, it is however very likely that different layers are dominating the emission in the jet and the counter-jet sides. To take this into account, we consider the difference between the apparent speed found in the jet and the speed measured in the counter jet. The counter-jet component $\mathrm{C} 1$ is detected at distances of $0.5-0.6$ mas from the core (see Fig. 10). In this region, we measure a jet to counter-jet intensity ratio $\mathcal{R}=9.5 \pm 1.5$. On the jet side, we can use a more robust velocity measurement obtained from the stacked cross correlation analysis discussed in Sect. 2.3. Between 0.5 and 1 mas from the core, we measure a jet apparent speed $\beta_{\mathrm{app}}^{\text {jet }}=0.21 \pm 0.04 c$ in the southern limb of the jet. Then, using Eq. (2), we obtain:

$\theta=17.2 \pm 3.3^{\circ}, \beta_{\text {jet }}=0.42 \pm 0.07, \beta_{\text {cjet }}=0.33 \pm 0.06$.

This estimate of the viewing angle is consistent with the constraint imposed by the maximum apparent speed $\beta_{\text {app }} \sim 6 c$ measured at HST- 1 which requires a viewing angle $\theta \lesssim 19^{\circ}$.

\subsection{Jet rotation}

\subsubsection{Evidence and estimation of the jet rotation}

The stacked cross correlation analysis has revealed substantial difference between the speeds measured in the northern and southern limbs of the jet (see Tables 2 and 3). At core separations of 0.5-1.0 mas, the significance of this difference reaches $\sim 4 \sigma$, while it is above $\sim 1.5 \sigma$ for both the fast and slow velocity components detected between 1 and 4 mas.

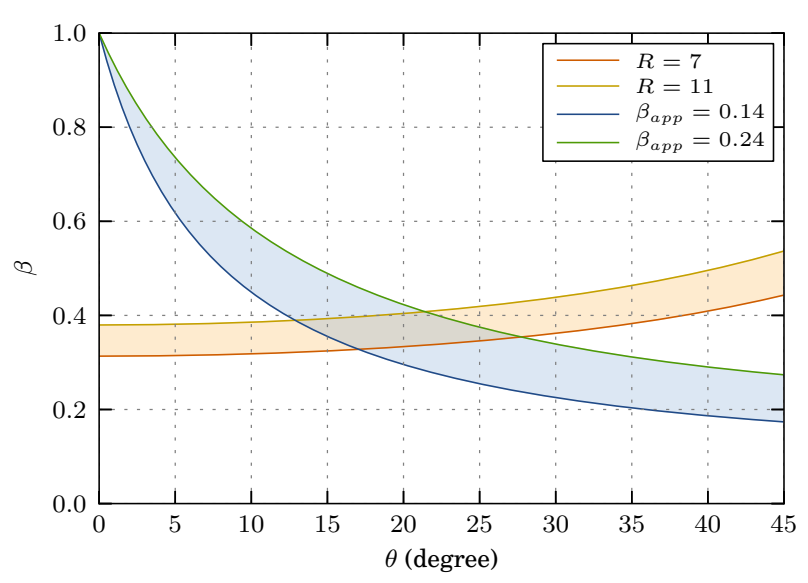

Fig. 11. Jet viewing angle constraints obtained from the minimum and maximum jet to counter jet intensity ratio $R$ and apparent velocity $\beta_{\text {app }}$ measured at core separations between 0.4 and 0.8 mas.

This difference can be explained naturally by invoking jet rotation. The intrinsic speed, $\beta$, of the flow can be decomposed into poloidal $\left(\beta_{\mathrm{p}}=\beta \cos \alpha_{\text {rot }}\right)$ and toroidal (or azimuthal, $\beta_{\phi}=\beta \sin \alpha_{\text {rot }}$ ) components, where $\alpha_{\text {rot }}$ is the angle between the intrinsic velocity and the jet axis. In this framework, for a clockwise rotation of the jet as viewed by the observer, the SSP components moving along the northern limb of the jet would be viewed at a larger viewing angle, increased by $\alpha_{\text {rot }}$, while in the southern limb, the effective viewing angle would be reduced by $\alpha_{\text {rot }}$. This effect would not apply to the central stream of the jet. However, since the velocities measured in the central stream can originate either from the near-side or the far-side of the jet, the effective viewing angle is increased by an angle $\psi$, which is zero when far-side and near-side of the jet contribute equally to the velocity detected in the central stream, and is bounded by the intrinsic instantaneous opening angle $\Theta$ of the jet. For the velocity vectors measured in the limbs, one need also to take into account the effect of the opening angle of the jet. The resulting geometrical setting is illustrated in Fig. 12.

Following this scheme, the effective viewing angles for the three streams in the jet can then be written as:

$$
\begin{aligned}
& \theta_{\text {north }}=\theta_{\text {edge }}+\alpha_{\text {rot }} \\
& \theta_{\text {south }}=\theta_{\text {edge }}-\alpha_{\text {rot }} \\
& \theta_{\text {center }}=\theta+\psi, \quad-\Theta \leq \psi \leq \Theta,
\end{aligned}
$$

where $\theta_{\text {edge }}$ is the edge viewing angle corrected for the opening angle of the jet:

$\cos \theta_{\text {edge }}=\frac{\cos \theta}{\left(\tan ^{2} \Theta+1\right)^{1 / 2}}$.

The intrinsic opening angle $\Theta$ can be estimated from the apparent opening angle measured in Fig. 6, using $\Theta=\Theta_{\text {app }} \sin \theta$.

Assuming that the flow has the same intrinsic velocity $\beta$ in the three regions, the rotation angle $\alpha_{\text {rot }}$ can be determined by solving the system of two equations:

$\left\{\begin{array}{l}\beta_{\text {app }}^{\text {north }}=\frac{\beta \sin \left(\theta_{\text {edge }}+\alpha_{\text {rot }}\right)}{1-\beta \cos \left(\theta_{\text {edge }}+\alpha_{\text {rot }}\right)} \\ \beta_{\text {app }}^{\text {south }}=\frac{\beta \sin \left(\theta_{\text {edge }}-\alpha_{\text {rot }}\right)}{1-\beta \cos \left(\theta_{\text {edge }}-\alpha_{\text {rot }}\right)} .\end{array}\right.$

If the velocity in the central stream is also considered, an additional equation can be written:

$\beta_{\text {app }}^{\text {center }}=\frac{\beta \sin (\theta+\psi)}{1-\beta \cos (\theta+\psi)}$. 


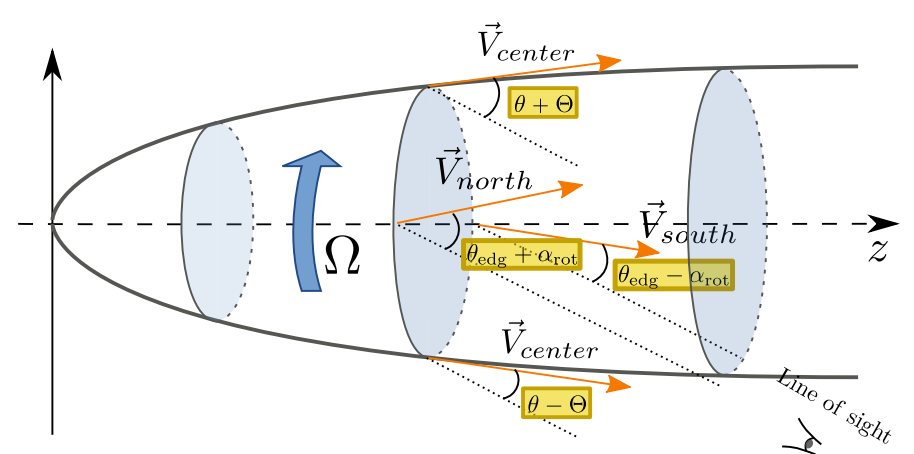

Fig. 12. Schematic illustration of the effect jet rotation has on the instantaneous viewing angles in the three jet streams. In a rotating outflow, the generic viewing angle of the jet axis, $\theta$ is modified by the angle, $\alpha_{\text {rot }}$, between the instantaneous velocity vector and the jet axis, and by the jet intrinsic opening angle $\Theta$, as described by Eqs. (5), (6).

Table 4. Parameters for jet rotation estimated in region A $(0.5$ mas $\leq$ $z_{\mathrm{obs}} \leq 1$ mas ) and region $\mathrm{B} 1$ mas $\leq z_{\mathrm{obs}} \leq 3$ mas for $\theta=18^{\circ}$.

\begin{tabular}{cccc}
\hline \hline Parameter & Region A & Region B, slow & Region B, fast \\
\hline$\alpha_{\text {rot }}\left[{ }^{\circ}\right]$ & $7.5 \pm 2.3$ & $-9.6 \pm 4.3$ & $4.5 \pm 2.5$ \\
$\beta_{\mathrm{p}}$ & $0.55 \pm 0.03$ & $0.54 \pm 0.15$ & $0.9245 \pm 0.003$ \\
$\beta_{\phi}$ & $0.08 \pm 0.02$ & $-0.10 \pm 0.05$ & $0.07 \pm 0.04$ \\
\hline
\end{tabular}

Notes. $\alpha_{\text {rot }}-$ viewing angle modification due to jet rotation; $\beta_{\mathrm{p}}, \beta_{\phi}-$ poloidal (axial) and toroidal (azimuthal) component of the flow speed.

A unique solution for Eqs. (7), (8) can be found with respect to the parameters $\alpha_{\text {rot }}, \beta$, and $\psi$ if the viewing angle of the jet, $\theta$, is known. Based on the analysis of the counter jet feature (see Sect. 4.2), a viewing angle $\theta=18^{\circ}$ can be adopted for this purpose. In the subsequent analysis, we will also test independently this hypothesis considering an equal contribution of the far-side and near-side of the jet in the central stream velocity $(\psi=0)$.

We use a least-square fitting technique to solve Eqs. (7), (8). Robust estimates of the fit uncertainties are obtained from a Monte Carlo simulation with 10000 trials using the input parameters drawn from Gaussian distributions with a mean and standard deviation provided by the fit value and the formal fit uncertainty for a given fit parameter. The resulting output distribution of the fit values also follows a Gaussian distribution, for each parameter of the fit. We therefore determine the uncertainty of the fit parameters by computing the standard deviation of this distribution.

The rotation parameters are calculated using the apparent velocities estimated from the SCC analysis in region A $(0.5$ mas $\leq$ $z_{\text {obs }} \leq 1$ mas), and independently for the slow and fast component identified in region $\mathrm{B}$ ( 1 mas $\leq z_{\mathrm{obs}} \leq 3$ mas). The results are summarized in Table 4, assuming a viewing angle $\theta=18^{\circ}$. Evidence for a clockwise rotation of the jet is found in region $\mathrm{A}$ with a $\sim 4 \sigma$ significance, and for the fast component of region $\mathrm{B}$. In this last case, we verify that $\psi$ is compatible with the inequality of Eq. (5): $\psi=-0.2 \pm 5^{\circ}$ while $\Theta=2.7^{\circ}$ at $z_{\text {obs }} \sim 2.5$ mas. Additionally we can obtain an independent estimate of the viewing angle of the jet $\theta$ assuming $\psi=0$ :

$\theta=19.2 \pm 3.7^{\circ}$

which supports very well our initial hypothesis. On the contrary, the slow component of region $\mathrm{B}$ is consistent with a counterclockwise rotation, which may indicate that this kinematic component reflects pattern motion rather than true physical rotation of the flow.

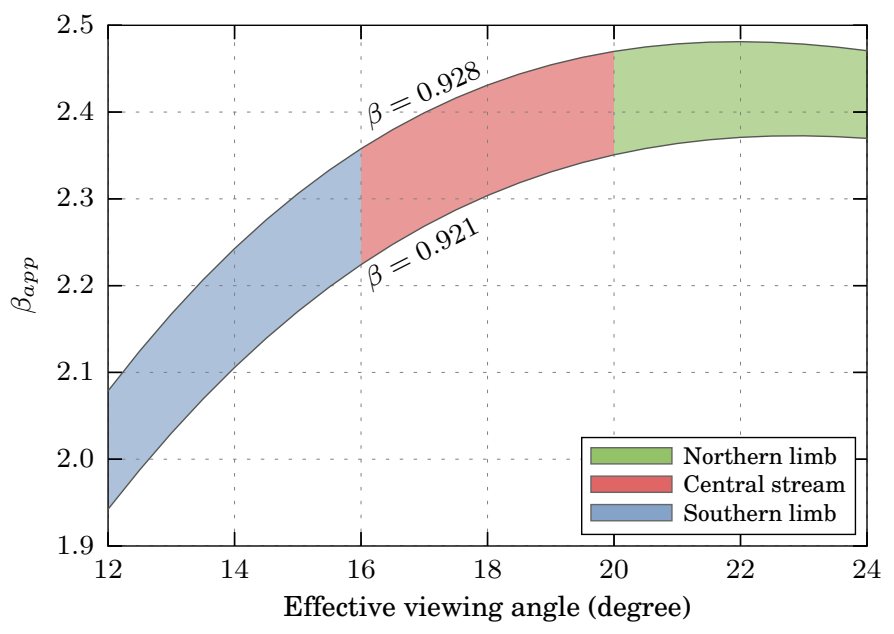

Fig. 13. Effect of jet rotation on the range of apparent velocities observed in the northern limb (green area), southern limb (blue area) and the central stream (red area) assuming the same intrinsic speeds in these three regions. With a viewing angle $\theta \sim 18^{\circ}$ and $\alpha_{\text {rot }} \sim 4^{\circ}$, we expect an effective viewing angle in the range $20-24^{\circ}$ for the northern limb stream and in the range $12-16^{\circ}$ for the southern limb stream.

Plasma instability can produce pattern motions with a substantial azimuthal velocity component which can both co-rotate and counter-rotate with the flow rotation (Bodo et al. 1996; Porth 2013). This might be the case for the slow component detected in region $\mathrm{B}$, which has already been associated with an instability pattern (see Sect. 4.1). A counterclockwise rotation of the instability pattern is also inferred for the jet in M 87 from the analysis of the internal structure of the flow on kiloparsec scales (Lobanov et al. 2003). We adopt therefore this explanation in our subsequent analysis of the physical conditions in the jet.

Jet rotation (irrespectively of its origin) can also explain the different broadening of the SCC peaks observed in Fig. 5 for the fast velocity component detected in Region $\mathrm{B}$. The observed gradual increase in the peak width going from the northern limb to the southern limb agrees well with the hypothesis of a different effective viewing angle in the three parts of the jet. For the same intrinsic speed in all three streams of the jet, Fig. 13 demonstrates that a smaller effective viewing angle (southern limb), will result in a larger range of apparent velocities, while on the contrary, a larger effective viewing angle (northern limb) will result in a smaller range of apparent velocities.

The smaller effective viewing angle to the flow in the southern limb relative to the northern limb resulting from rotation would result in differential Doppler boosting. Instabilities and the potential slower outer layer make it difficult to accurately measure jet rotation from the brightness ratio between the two limbs. However, the mean brightness ratio between the northern and southern limbs is 0.56 , and close to the 0.4 value predicted to accompany our estimated fast layer jet rotation.

\subsubsection{Interpretation of the jet rotation}

If the azimuthal components of the jet velocity determined above reflects physical rotation of the jet, they can be related to specific mechanisms of formation and acceleration of relativistic flows in AGN. In the context of an MHD jet launched by magnetocentrifugal forces, the initial toroidal velocity is associated either with the Keplerian speed at the launching location in the accretion disk (Blandford \& Payne 1982, BP mechanism) or 

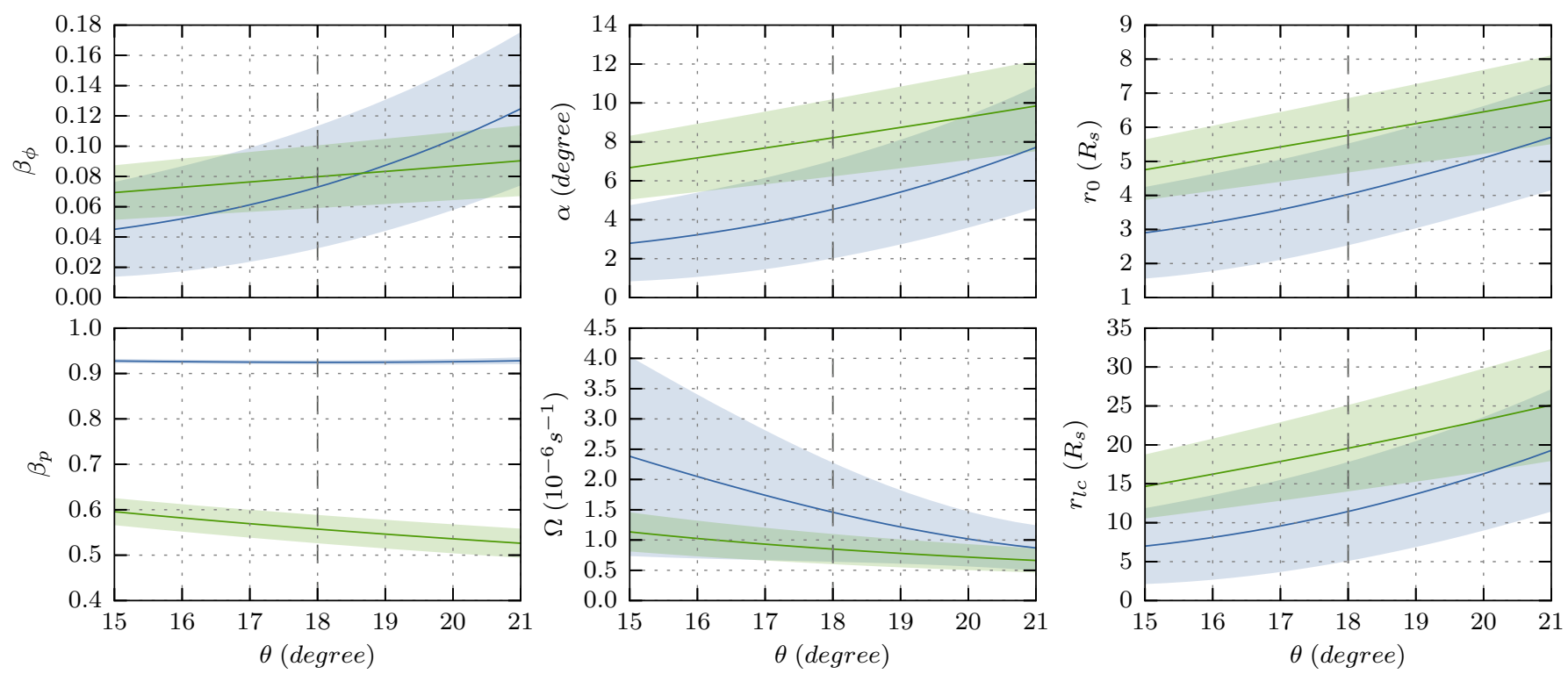

Fig. 14. Parameters for jet rotation obtained in Region A (green) and the fast component in Region B (blue) evaluated over a range of jet viewing angles. The shaded region corresponds to one sigma uncertainty.

with the spin of the central engine (Blandford \& Znajek 1977, BZ mechanism). Conservation of angular momentum will result in a slow decrease in the toroidal velocity beyond the light cylinder (Vlahakis 2015). Observational signature of jet rotation has been previously reported in jets from young stellar objects (YSO), based on spectroscopic radial velocity analysis (e.g., Anderson et al. 2003; Choi et al. 2011), and has been successfully associated with an MHD process, giving a constraint on the jet launching location. Jet rotation has so far not been directly detected in AGN jets, with the reported rotational speeds clearly related to the rotation of patterns inside the flow (e.g., Lobanov et al. 2003; Agudo et al. 2007, 2012; Cohen et al. 2015) resulting from plasma instability in the flow. If our measured rotation is associated with conserved angular momentum in the jet and if the jet is launched from the accretion disk via the BP mechanism, its direction should be the same as the rotational direction of the disk itself. Using spectroscopic HST measurements, Harms et al. (1994) found that the ionized gas associated with the accretion disk in M 87 rotates in a clockwise direction. This is the observed rotation direction found in region $\mathrm{A}$ and for the fast component in region $\mathrm{B}$. It is also interesting to note that the toroidal velocity measured closer to the central engine is greater than that measured farther away, which is what the conservation of angular momentum predicts. We will now try to associate this observed rotation with the MHD properties of the flow, following Anderson et al. (2003).

In the approximation of a cold steady MHD jet, the equations of conservation of total (magnetic + matter) specific energy $\mu$ and angular momentum $L$ can be combined, eliminating the toroidal magnetic field $B_{\phi}$ and the distribution of mass flux at the inlet of the flow $\eta$, and yielding the jet specific momentum $J$ :

$$
\begin{aligned}
& \mu=\gamma-\frac{r \Omega B_{\phi}}{c \eta} \\
& L=r c \gamma \beta_{\phi}-\frac{c r B_{\phi}}{\eta} \\
& J=\mu c^{2}-\Omega L=\gamma c^{2}-\Omega r c \gamma \beta_{\phi} .
\end{aligned}
$$

The jet specific momentum, $J$, is conserved along any particular field line and can be used to obtain an expression for the angular
Table 5. Estimated angular velocity and launching location for Region A and for the fast component in Region B, with $\theta=18^{\circ}$.

\begin{tabular}{ccc}
\hline \hline Parameter & Region A & Region B, fast \\
\hline$\Omega\left[10^{-6} \mathrm{~s}^{-1}\right]$ & $0.9 \pm 0.3$ & $1.5 \pm 0.8$ \\
$r_{0}\left[R_{\mathrm{s}}\right]$ & $5.7 \pm 1.1$ & $4.0 \pm 1.5$ \\
$r_{\mathrm{lc}}\left[R_{\mathrm{s}}\right]$ & $19 \pm 5$ & $11 \pm 6$ \\
\hline
\end{tabular}

velocity, which is also a conserved quantity along a given field line:

$\Omega=\frac{c}{r \beta_{\phi}}\left(1-\frac{J}{c^{2} \gamma}\right)$.

At the origin of the outflow, we can assume that $\beta_{\phi}$ is well below the speed of light $\left(\Omega r_{0} \ll c, \beta_{\phi \text {,in }} \ll 1\right)$, which gives $J \simeq \gamma_{\text {in }} c^{2} \simeq$ $c^{2}$. Knowing the toroidal velocity and Lorentz factor of the flow, Eq. (11) can be used to estimate the angular velocity and the associated light cylinder radius $\left(r_{\mathrm{lc}}=c / \Omega\right)$. Assuming Keplerian rotation in the accretion disk, we can also obtain the launching location of a field line:

$r_{0}=\left(\frac{G M_{\mathrm{BH}}}{\Omega^{2}}\right)^{1 / 3}$,

with $M_{\mathrm{BH}}$ denoting the mass of the central engine.

The resulting parameters obtained for region $\mathrm{A}$ and for the fast component in region B are summarized in Table 5 for a viewing angle of $18^{\circ}$. For completeness, the respective rotation parameters and angular velocity estimates for viewing angles between $15^{\circ}$ and $21^{\circ}$ are also provided in Fig. 14. Continuity of the flow between regions $A$ and $B$, which can also be inferred from the VLBA images (see Fig. 2), indicates that both these regions can represent the same field line, and hence should yield similar underlying rotational properties of the flow. Indeed, the estimates listed in Table 5 for the two jet regions agree within the uncertainties, and a weighted average of the two measurements gives

$\Omega=1.1 \pm 0.3 \times 10^{-6} \mathrm{~s}^{-1}$
$r_{0}=4.8 \pm 0.8 R_{\mathrm{s}}$. 
Several potential difficulties in invoking jet rotation in the interpretation of some observed properties in YSO outflows have been discussed in the literature. Numerical simulations have shown that several disruptive effects, for instance shocks (Fendt 2011) or plasma instability (Staff et al. 2015) can indeed affect the rotational signature from YSO jets. These effects should be mitigated in our case, as our rotation measurements also include a fast component velocity which should be related to the inner layers of the jet and thus less affected by $\mathrm{KH}$ instability operating near the jet surface (however a body mode of the KH instability or current-driven instability could still have an impact on the observed jet rotation). We remark also that no re-collimation shocks are observed in the VLBA images of the M 87 jet and that the jet continues to be straight and steady up to Knot A at a de-projected distance of several kiloparsecs. We finally note that the location of the jet launching region that we derived from the angular velocity measurement is in the inner part of the accretion disk close to the innermost stable circular orbit (ISCO) of the black hole, which agrees well with the predication from numerical simulations of magnetized relativistic jets (Meier et al. 2001; Komissarov et al. 2007).

\subsection{Jet collimation and acceleration}

Analytical and numerical simulation MHD work has shown that the processes of jet collimation and acceleration are related and occur on the same spatial scales in relativistic flows. Most of the flow acceleration might result from the so-called "magnetic nozzle" effect (Li et al. 1992; Vlahakis 2015). This effect is at work whenever the flow collimates so that the distance between streamlines increases faster than the radius of the flow. This results in a small deviation of the local poloidal magnetic field from the mean magnetic field and this drives the acceleration. Since this deviation is small, the fluid is only slowly accelerated by a small residual force and the acceleration zone can extend over a large distance. Both numerical simulations and observational evidence suggest that jet acceleration continues up to distances of $10^{4}-10^{5} R_{\mathrm{s}}$ from the central engine (Vlahakis \& Königl 2004; Lobanov \& Zensus 1996, 1999; Homan et al. 2015; Lee et al. 2008, 2016).

The ideal MHD equations describing plasma jets may be written as a set of two non-linear differential equations describing the equilibrium of forces perpendicular (Grad-Shafranov or transfield equation) and parallel (Bernoulli equation) to the magnetic surface (Camenzind 1986; Fendt \& Ouyed 2004). A complete analytic solution of these equations can be obtained only in specific, restricted cases, (e.g., self-similar solutions). The forcefree approximation is often used to describe jets in the near zone, inside the light cylinder ( $r \lesssim r_{\mathrm{lc}}$; e.g., Blandford \& Znajek 1977). At larger radial separations, different sets of approximations may need to be used.

In a recent paper, Lyubarsky (2009) obtained asymptotic solutions for the case of a cold, ideal, Poynting flux dominated $(\sigma \gg 1)$ MHD jet in the far-zone $\left(r \gg r_{\text {lc }}\right)$. In these solutions, different sets of approximations are used, depending on the decay of the external pressure confining the jet. In AGN, this confinement can be achieved through a variety of forces including gas pressure from the ambient medium, ram pressure from the outer, subrelativistic wind and the stress of a magnetic field anchored in the disk. In general, evolution of the confining pressure can be approximated by a power law:

$P_{\text {ext }}=P_{0} z^{-\kappa}$,
Lyubarsky (2009) found two different collimation and acceleration regimes for the jet, depending on specific ranges of the power index $\kappa$.

If $\kappa<2$, the jet is in the so-called equilibrium regime. The residual of the hoop stress and the electric force are counterbalanced by the pressure of the poloidal magnetic field so that, at any distance from the source, the structure of the flow is the same as the structure of an appropriate cylindrical equilibrium configuration. The jet shape is given by a power law $r \propto z^{k}$, with $k=\kappa / 4$ converging to a cylindrical shape at large distance. The acceleration is linear, meaning that it is proportional to the jet radius: $\gamma \propto r \propto z^{k}$.

If $\kappa>2$, the jet is at first in a regime similar to the equilibrium regime described above. The jet shape is parabolic $r \propto z^{k}$ and the acceleration is linear. At some distance, the pressure of the poloidal magnetic field becomes small, and the flow could be viewed as a composition of coaxial magnetic loops. This regime is called non-equilibrium. The flow reaches ultimately a conical shape and the acceleration is determined by the curvature of the flow, $\gamma \propto z^{(\kappa-2) / 2}$

We found in Sect. 2.4 that the evolution of the cylindrical cross-section of the jet in M 87 is described by a power law with an index $k \sim 0.56-0.60$. Asada \& Nakamura (2012) found that the M87 jet reaches a conical shape at kiloparsec scales, similarly to the $\kappa>2$ case described above. To determine the acceleration profile, we compute the Lorentz factors for the velocity field obtained from WISE analysis at $7 \mathrm{~mm}$ (Sect. 2.1) and $2 \mathrm{~cm}$ (Sect. 3), using a viewing angle $\theta=18^{\circ}$. We complement the radio measurements with the speeds determined from optical and radio images of the kiloparsec-scale jet in M 87 (Biretta et al. 1999; Cheung et al. 2007; Giroletti et al. 2012; Asada et al. 2014). The combined evolution of the Lorentz factor plotted in Fig. 15 indicates two different regimes for the acceleration. The acceleration is at first linear with $\gamma \propto r \propto z^{0.58}$ up to $z \sim 8$ mas $\left(\sim 10^{3} R_{\mathrm{s}}\right)$, and then it becomes weaker, with $\gamma \propto z^{0.16}$, agreeing well with the measurements obtained at larger scales, up to the knot HST-1. The jet appears to enter the non-equilibrium regime $(\kappa>2)$ on these scales. One must however be cautious about this interpretation. The scaling relations obtained for the $\kappa>2$ case are obtained for a conical jet. However the jet in M 87 transits to a conical shape only at $z \sim 10^{3}$ mas (Asada \& Nakamura 2012). An alternative explanation for this slower acceleration can be offered by an early saturation of Poynting flux conversion. In this case, the conversion should be nearly complete at $z \sim 10^{3} R_{\mathrm{s}}$, and acceleration would then continue slowly until full conversion is achieved.

In the following, the acceleration and collimation of the jet in M 87 is tested against the magnetically accelerated jet model. Two different methods are used to solve the MHD equations. The first method is based on the analytical approximations obtained by Lyubarsky (2009) which describes Poynting flux dominated flows and is mainly used to test the initial acceleration phase. A second method, that relaxes the assumption of a Poynting dominated jet, is used to investigate the second acceleration phase, the saturation of Poynting flux conversion and the transition to a kinetically dominated jet.

The velocity profile corresponding to the fast velocity component of the flow, which has been associated with the sheath of the jet in Sect. 4.1, is obtained by computing the mean of the fastest $10 \%$ of the speeds measured within individual bins of 0.1 mas in size. To improve the robustness of the velocity determination, resampling is done using bins of 0.2 mas for $0.4 \leq z_{\text {obs }}<2$ mas, 0.3 mas for $2 \leq z_{\text {obs }}<4$ mas and finally 0.6 mas for $z_{\text {obs }} \geq 4$ mas. The result is plotted in Fig. 16. We 


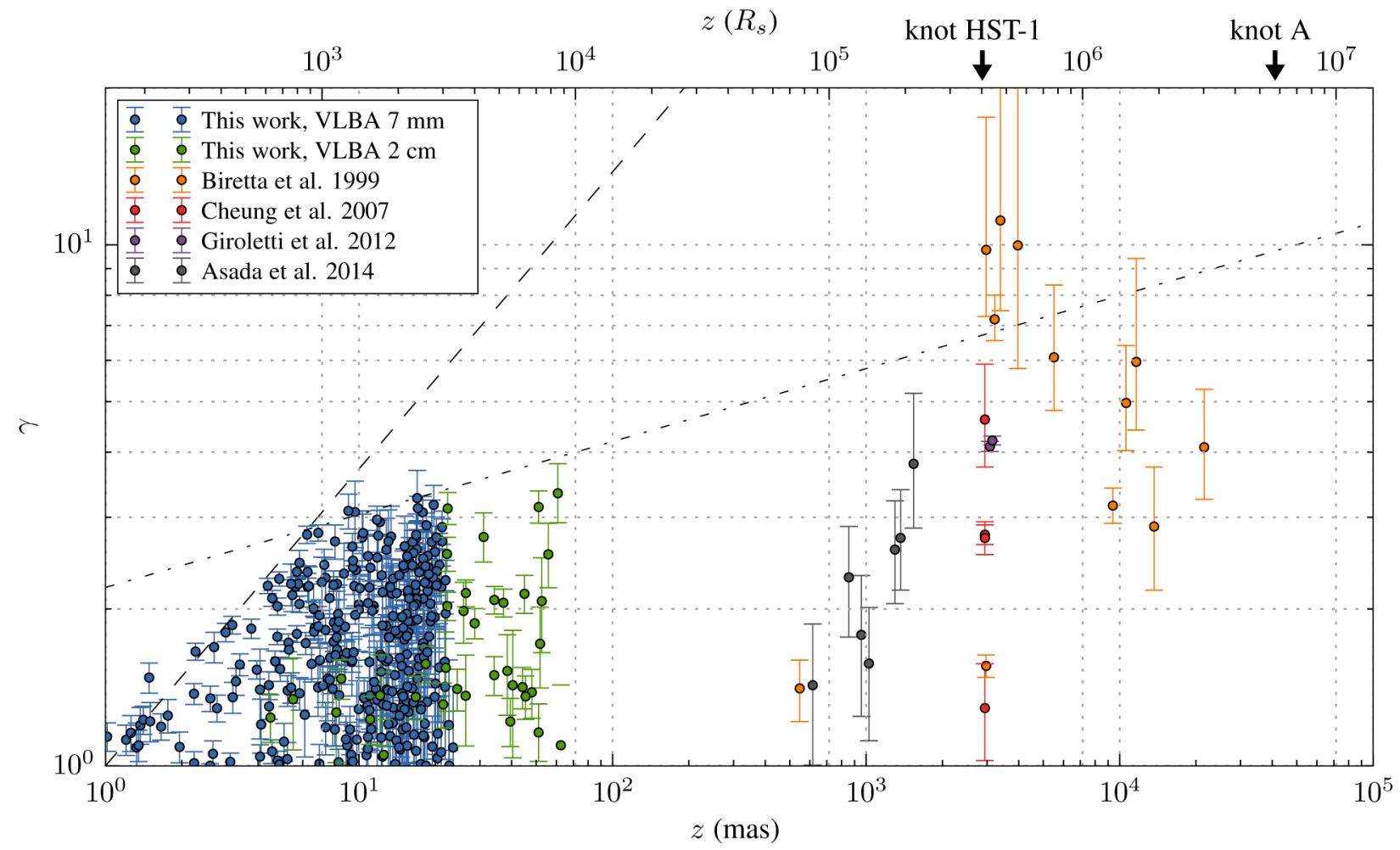

Fig. 15. Acceleration profile of the jet in M 87. We plot the Lorentz factor measured from WISE analysis of VLBA images at $7 \mathrm{~mm}$ (blue), $2 \mathrm{~cm}$ (green), from Cheung et al. (2007) using VLBA at $1.7 \mathrm{GHz}$ (red), from Giroletti et al. (2012) and Asada et al. (2014) using EVN at 1.7 GHz (magenta and gray respectively), and from optical images using HST (Biretta et al. 1999; orange). The Lorentz factor and de-projected $z$ axis are computed assuming $\theta=18^{\circ}\left(z \sim 3.2 z_{\text {obs }}\right)$. Two regimes are found with a linear acceleration up to $z \sim 10^{3} R_{\mathrm{s}}$, followed by a slow acceleration up to HST-1

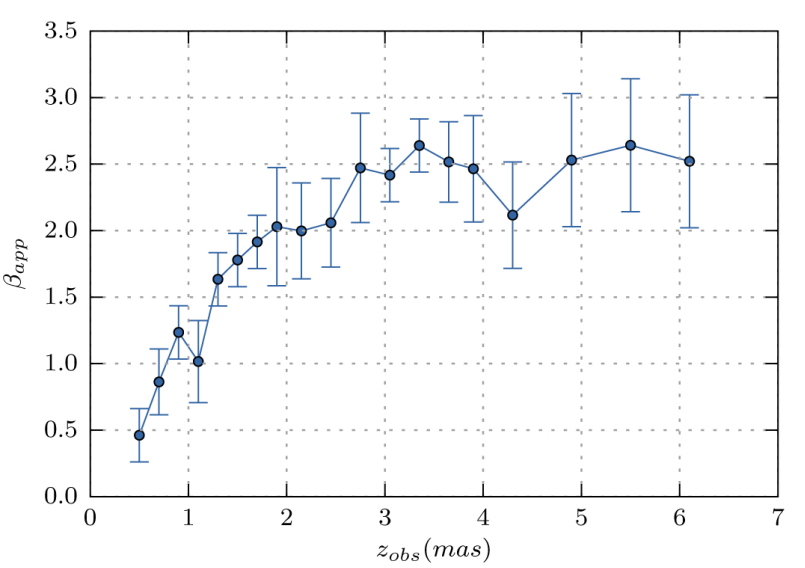

Fig. 16. Evolution of the apparent velocity with distance from the core of the fast velocity component in the jet sheath.

ascribe these measured velocities to a single field line (located on a single magnetic flux surface) with a profile corresponding to the collimation profile of the sheath as determined in Sect. 2.4. Under this assumption, and assuming $\Omega=$ const along this field line, we can conveniently use variables scaled to the light cylinder radius, $R=\Omega r / c$ and $Z=\Omega z / c$.

\subsubsection{Asymptotic relations in the far-zone Poynting dominated approximation}

In the ideal cold MHD approximation, one can further simplify the solutions by considering the additional approximation of a
Poynting dominated jet $(\sigma \gg 1)$ in the far-zone $(R \gg 1, \gamma \gg$ 1) (Lyubarsky 2009).

In the equilibrium case and for $\kappa>2$ one can obtain the following relations for the jet shape:

$$
\begin{aligned}
& R=\left(\frac{3 Z^{\kappa}}{\epsilon_{\mathrm{p}}}\right)^{1 / 4} f \\
& f=\sqrt{\frac{2-\kappa}{\pi}}\left[\frac{1}{C_{1}} \cos ^{2} w+C_{1}\left(C_{2} \cos w+\frac{\pi}{2-\kappa} \sin w\right)^{2}\right]^{1 / 2} \\
& w=\frac{2 \sqrt{\epsilon_{\mathrm{p}}}}{2-\kappa} Z^{1-\kappa / 2}-\frac{4-\pi}{2-\kappa} \frac{\pi}{4},
\end{aligned}
$$

with $\epsilon_{\mathrm{p}}$ describing the ratio of the plasma pressure to the magnetic pressure:

$\epsilon_{\mathrm{p}}=\frac{6 \pi p_{0}}{B_{0}^{2}}$,

where $B_{0}$ and $p_{0}$ are the characteristic magnetic field and the external pressure at the light surface, respectively. In the nonequilibrium case, the jet shape is asymptotically conical.

Evolution of the Lorentz factor can also be obtained, depending on the acceleration regime:

$\gamma= \begin{cases}R & \text { (equilibrium), } \\ \frac{Z^{(\kappa-2) / 2}}{\sqrt{\beta} \Theta_{\text {app }}^{\text {break }}} & \text { (non-equilibrium), }\end{cases}$

where $\Theta_{\text {app }}^{\text {break }}$ is the local opening angle at the transition between the two regimes. From Fig. 16, we estimate that this transition 
Table 6. Best fit parameters reproducing the observed acceleration and collimation using asymptotic relations in the far-zone of the Poynting flux dominated approximation.

\begin{tabular}{lr}
\hline \hline Parameter & Best fit \\
\hline$z_{\text {obs }}^{\text {break }}[$ mas $]$ & 1.7 (fixed) \\
$\Theta_{\text {app }}^{\text {break }}\left[{ }^{\circ}\right]$ & 10.5 (fixed) \\
$k$ & $0.588 \pm 0.005$ \\
$\Omega\left[10^{-6} \mathrm{~s}^{-1}\right]$ & $0.86 \pm 0.02$ \\
$\epsilon_{\mathrm{p}}$ & $79 \pm 12$ \\
$\theta\left[{ }^{\circ}\right]$ & $19 \pm 4$ \\
$C_{1}$ & $0.10 \pm 0.03$ \\
$C_{2}$ & $-1 \pm 2$ \\
$a_{\gamma}$ & $0.63 \pm 0.13$ \\
$\phi_{\gamma}\left[{ }^{\circ}\right]$ & $35 \pm 7$ \\
\hline
\end{tabular}

occurs in the jet in M 87 around $z_{\text {obs }}^{\text {break }} \sim 1.7$ mas, which, in return, corresponds to $\Theta_{\text {app }}^{\text {break }} \sim 10.5^{\circ}$.

The Lorentz factor and the collimation profile are fit simultaneously using the Levenberg-Marquardt least-squares minimization routine. Uncertainties in the fit parameters are determined from the standard error obtained from the estimated covariance matrix. The collimation profile is modeled using the relation from Eq. (15). The two phases of the acceleration, before and after $z_{\text {obs }}^{\text {break }}$, are modeled using the relations from Eq. (17) for the equilibrium and non-equilibrium case respectively.

Some modifications to the Lorentz factor are required for the fit to converge. An additional scaling, $\alpha_{\gamma}$, was introduced modifying the Lorentz factor so that: $\gamma^{\star}=a_{\gamma} \gamma$, and the Lorentz factor was phase shifted along the axial coordinate of the jet by $w_{\gamma}^{\star}=w+\phi_{\gamma}$, in order to be able to account for the fact that the maxima and minima found in the collimation profile do not correspond exactly to the maxima and minima found in the Lorentz factor profile. The Lorentz factor for the nonequilibrium case was obtained by Lyubarsky (2009) considering a non-oscillating conical jet shape. Figure 16 shows that, even after $z_{\text {obs }}^{\text {break }}$, the Lorentz factor is still oscillating. We account for this observation by extending, in our model, the oscillation to the non-equilibrium case, so that $\gamma_{\mathrm{ne}}^{\star}=a_{\gamma} \gamma_{\mathrm{ne}} f$.

All parameters are allowed to vary freely, including the viewing angle $\theta$ and the angular velocity $\Omega$. The best fit obtained under these conditions has a reduced $\chi^{2}=0.24$. In detail, this corresponds to $\chi_{r}^{2}=0.22$ and $\chi_{\gamma}^{2}=0.34$ for the collimation and Lorentz factor fit respectively. We recall that $\chi_{r}^{2}=0.55$ was found by modeling the collimation profile as a simple power law. This indicates an improvement, by a factor of $\sim 2$, of the quality of the fit provided by the Poynting flux approximation for the jet collimation profile. We note that the relatively small values obtained for the reduced chi-squared is most probably a consequence of our conservative choice for the errors in the jet radius and jet velocity.

The best fit is plotted in Fig. 17, and the derived parameters of the model are summarized in Table 6 . It is remarkable that the viewing angle and the angular velocity agree, within the uncertainties, with the respective values found from the analysis of the counter jet (Sect. 4.2) and the jet rotation (Sect. 4.3). The oscillations in both the jet width and the Lorentz factor that we discussed in Sect. 2.4 are also particularly well reproduced by the model, and if we extend this solution, we find that the subsequent Lorentz factor located at $z_{\mathrm{obs}}=6.8$ mas and $z_{\mathrm{obs}}=17.2 \mathrm{mas}$, coincide with the two regions of maximum apparent velocities

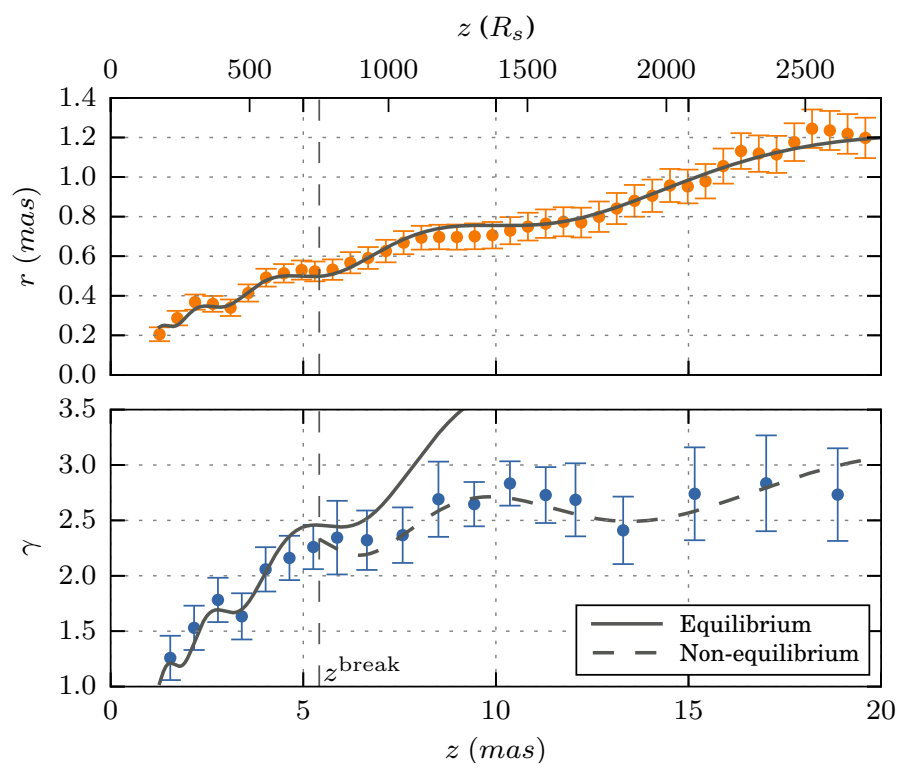

Fig. 17. Best fit for the observed acceleration (blue) and collimation (orange) of the jet in M 87. The viewing angle and angular velocity are independently determined from the fit. Parameters of the fit model are listed in Table 6.

identified in our WISE analysis of the MOJAVE observations (Sect. 3). Komissarov et al. (2015) have interpreted these oscillations as a standing magneto-sonic wave bouncing across the jet.

There are several reasons why a phase shift, $\phi_{\gamma}$, and scaling factor, $\alpha_{\gamma}$, are required in our modeling. A possible reason for the phase shift $\phi_{\gamma}$ is that the measured Lorentz factor may come from a streamline slightly inside the measured jet shape. In this case the maxima in the Lorentz factor will be offset from the maxima in the jet width, with a shift proportional to the jet radius (Norman et al. 1982). The need for the scaling factor, $\alpha_{\gamma}$, may be found in the assumption made by Lyubarsky (2009) of a constant angular velocity for all field lines. This assumption is usually valid only for a jet launched from the magnetosphere. For the case of disk launching, we expect differential rotation and Tchekhovskoy et al. (2008) found $\gamma<R$ under these conditions. We recall here also that relations were derived using assumptions, specifically $R \gg 1$ and $\gamma \gg 1$, which are only partially valid in our case.

While the Lorentz factor of the second acceleration regime is well modeled by the non-equilibrium case, it is not associated with transition to the conical jet shape that one should expect theoretically. The apparent opening angle at the location where the acceleration regime changes is $\Theta_{\text {app }}^{\text {break }} \sim 10.5^{\circ}$, and therefore additional collimation is necessary to achieve the final apparent opening angle $\Theta_{\mathrm{app}}^{\mathrm{kpc}} \sim 3.2^{\circ}$ observed at kiloparsec scales. Early saturation of the Poynting flux could also result in a quenched acceleration, and would break the assumptions of a Poynting dominated flux used in this modeling. We will now investigate this possibility by using another method of solving the MHD equations.

\subsubsection{Wind solutions}

Solving simultaneously the full transfield and the Bernoulli equations is only possible in a restrictive number of cases. The transfield equation will determine the shape of the field lines while the Bernoulli equation governs the acceleration of the flow. It is however possible to solve only the Bernoulli 
equation by assuming a specific jet shape (e.g., Camenzind 1986; Fendt \& Ouyed 2004; Toma \& Takahara 2013). The resulting solution is called a wind solution. It is not strictly an exact MHD solution, and one should be careful not to over-interpret it. However it can be used in some specific cases, for example, for investigating the acceleration efficiency, or in our case, the acceleration profile.

In the context of a cold ideal MHD jet, the poloidal and toroidal velocities can be written as follows:

$$
\begin{aligned}
& \beta_{\mathrm{p}}=\frac{R^{2} B_{\mathrm{p}}(R)}{\eta(\mu-\gamma)}\left(1-\frac{1}{R^{2}}\left(1-\frac{\gamma_{\text {in }}}{\gamma}\right)\right) \\
& \beta_{\phi}=\frac{1}{R}\left(1-\frac{\gamma_{\text {in }}}{\gamma}\right),
\end{aligned}
$$

where $B_{\mathrm{p}}$ is the poloidal magnetic field, $\mu$ is the total (magnetic + matter) specific energy, $\gamma_{\text {in }}$ and $\eta$ are the Lorentz factor and the distribution of mass flux at the inlet of the flow, respectively. The quantity $\eta$ is a field line constant and it is given by the following equation:

$\eta=\frac{4 \pi \gamma \rho_{0} \beta_{\mathrm{p}} c^{2}}{B_{\mathrm{p}}}$,

with $\rho_{0}$ describing the rest-mass density. We also define the ratio of the Poynting to the matter energy flux, also called magnetisation parameter, as:

$\sigma=\frac{\mu-\gamma}{\gamma}$

The Bernoulli equation is obtained from:

$\beta_{\mathrm{p}}^{2}+\beta_{\phi}^{2}+\frac{1}{\gamma^{2}}=1$

Substituting $\beta_{\mathrm{p}}$ and $\beta_{\phi}$ using Eq. (18), one can reduce the Bernoulli equation to a quartic equation for $\gamma$. To solve it, one has to prescribe a model for the poloidal magnetic field $B_{\mathrm{p}}$, which turns into a model for the flux function.

Toma \& Takahara (2013) recently derived a set of models for $B_{\mathrm{p}}$ based on a realistic flux function $\Psi(r, z)$ which describes parabolic field lines appropriate for M 87:

$Z+\zeta(\psi)=A(\psi)(R-\varpi(\psi))^{a(\psi)}$,

where $\psi=\Psi / \Psi_{0}$ and $\Psi_{0}$ is the total magnetic flux, and $\zeta(\psi), A(\psi), \varpi(\psi)$, and $a(\psi)$ are arbitrary functions of $\psi$ (Toma \& Takahara 2013). The poloidal magnetic flux is then obtained recalling that $B_{\mathrm{p}}=(\nabla \Psi \times \phi) / r$, and this yields

$$
\begin{aligned}
B_{\mathrm{p}}(R)= & \frac{\Psi_{0}}{R^{2}}\left(\frac{\zeta^{\prime}}{Z+\zeta}-\frac{A^{\prime}}{A}-a^{\prime} \ln (R-\varpi)+\frac{a \varpi^{\prime}}{R-\varpi}\right)^{-1} \\
& \times \frac{R}{R-\varpi} \sqrt{\left(\frac{R-\varpi}{Z-\zeta}\right)^{2}+a^{2}},
\end{aligned}
$$

with primes denoting derivatives of the respective functions over $\psi$.

At $r \lesssim r_{\text {lc }}$, the true shape of field lines will probably deviate from the shape derived from Eq. (22) where field lines are anchored into the accretion disk or the magnetosphere. However, the approximation should be valid in the region we are investigating.

In our case of interest, associating the jet sheath with the outer poloidal field line $(\psi=1)$ and using $\theta=18^{\circ}$ and $\Omega=10^{-6}$,

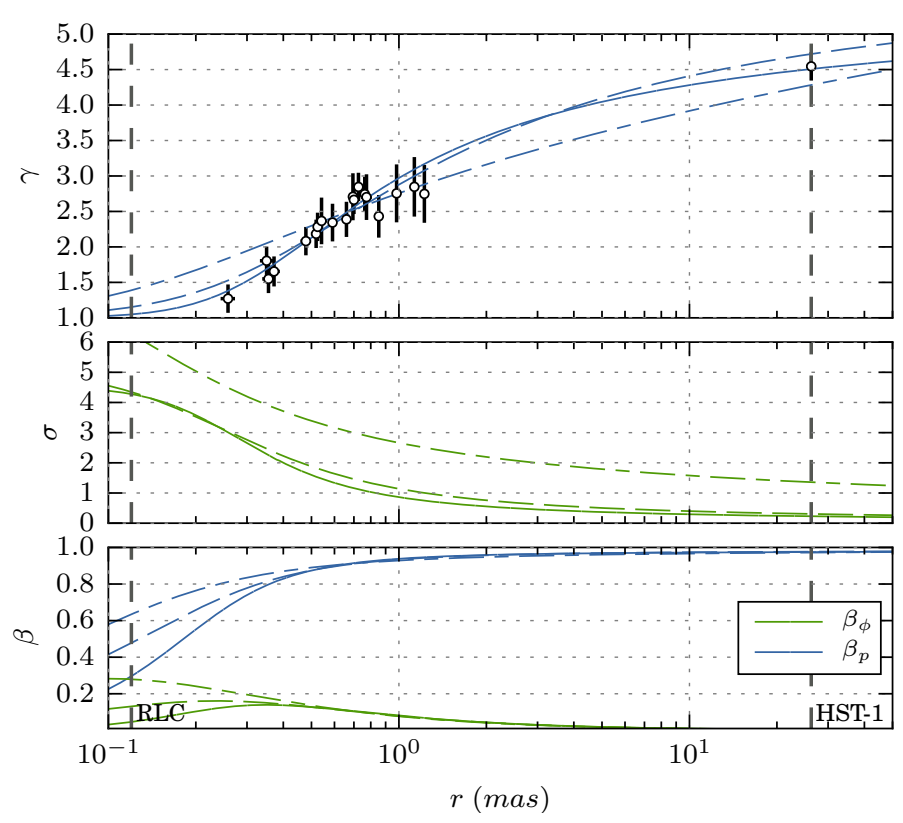

Fig. 18. Wind solution for model 1 (solid line), model 2 (dashed line) and model 3 (long dash short dash line) for the flux function that best reproduces the observed acceleration (black points). The upper and middle panels show the total energy (blue) and magnetisation parameter (green). The lower panel shows the toroidal (green) and poloidal (blue) velocity.

we have $\zeta=\varpi=0, A=3.7$ and $a=1 / k=1.73$, corresponding to the parabolic shape obtained by Asada \& Nakamura (2012). We also adopt $\gamma_{\text {in }} \rightarrow 1$.

With the assumptions specified above, the Bernoulli equation still contains 7 unknowns: the total specific energy $\mu$, the distribution of mass flux at the inlet $\eta$, the total magnetic flux $\Psi_{0}$, and four parameters related to the geometry of the poloidal field lines $\left(a^{\prime}, A^{\prime}, \zeta^{\prime}\right.$ and $\left.\varpi^{\prime}\right)$. We can however isolate some parameters and treat different cases separately. We define three models corresponding to different configurations of the flux function:

Model 1: $a^{\prime} \neq 0, A^{\prime}=0, \zeta^{\prime} \neq 0, \varpi^{\prime}=0$,

Model 2: $a^{\prime} \neq 0, A^{\prime}=0, \zeta^{\prime}=0, \varpi^{\prime} \neq 0$,

Model 3: $a^{\prime} \neq 0, A^{\prime} \neq 0, \zeta^{\prime}=0, \varpi^{\prime}=0$.

The Bernoulli equation has two singularities at the Alfvénic point (AP) and at the fast magnetosonic point (FMP). The wind solution in which $\gamma$ is growing toward infinity has to pass through both the AP and the FMP. The Bernoulli equation, being quartic for $\gamma$, admits four roots. To be valid, the wind solution needs to pass smoothly from one root to the other crossing the singularities. This provides an additional constraint on the solution, and for a given $B_{\mathrm{p}}(R)$ and $\eta$, a unique value of $\mu$ corresponds to a valid wind solution. Further examining the Bernoulli equation, we find that $\Psi_{0}$ and $\eta$ can not be separated, and their ratio must therefore be treated as a single variable. Hence we end up with only three free variables for the three models described above.

A Nelder-Mead least-squares minimization routine is used to fit the Bernoulli equation to the measured Lorentz factor evolution. Within each iteration of the fitting algorithm, $\mu$ is determined so as to maintain a valid wind solution.

Satisfactory fits were obtained for Models 1 and 2, however we could not obtain a reasonable fit using Model 3. In order still to be able to analyze the impact of the parameter $A^{\prime}$ on the solution, we assigned a small non-zero value to $\varpi^{\prime}$ in this model 
Table 7. Parameters for the wind solution that reproduce the observed acceleration for the three flux function models.

\begin{tabular}{lrrr}
\hline \hline Parameter & Model 1 & Model 2 & Model 3 \\
\hline$\mu$ & 5.5 & 6.2 & 10.1 \\
$L\left(\times 10^{23}\right)[\mathrm{SI}]$ & 4.1 & 4.7 & 8.2 \\
FMP $\left[R_{\mathrm{lc}}\right]$ & 3.23 & 3.23 & 3.65 \\
AP $\left[R_{\mathrm{lc}}\right]$ & 0.92 & 0.92 & 0.95 \\
$B_{0} / \eta[\mathrm{SI}]$ & 1.5 & 2.9 & 7.8 \\
\hline$\chi^{2}$ & 0.41 & 0.62 & 1.6 \\
\hline
\end{tabular}

Notes. $B_{0}=B_{\mathrm{p}}\left(r_{\mathrm{lc}}\right)-$ characteristic magnetic field at the light cylinder;

$\left(\varpi^{\prime}=0.05\right)$. The results for the three models are summarized in Table 7 and corresponding wind solutions are plotted in Fig. 18.

Figure 15 suggests that the velocities obtained for knot HST- 1 and from the $43 \mathrm{GHz}$ VLBA images originate from the same field line. To test this hypothesis we also include to the fit the speed measured at HST-1 by Giroletti et al. (2012), assuming $r_{\mathrm{HST}-1}=26$ mas (Asada $\&$ Nakamura 2012). This addition does not affect the reduced $\chi_{2}$ of the fit, thus supporting our hypothesis.

Models 1 and 2 best reproduce the measured acceleration. Jet parameters derived with these models are also similar. In Model 3, a higher value of $\mu$ was required, and the resulting conversion from Poynting flux to kinetic energy is slower. In all three cases, the Alfvénic point is found to be located close to the light cylinder, in agreement with exact self-similar solutions (Vlahakis \& Königl 2004). The fast magnetosonic point is located at $r \sim 0.4$ mas, corresponding to $z \sim 1 \mathrm{mas}$, and in agreement with the expectation that $\gamma_{\mathrm{FMP}} \simeq \mu^{1 / 3}$.

A true solution of the Bernoulli equation for the jet in M 87 would most likely be represented by a combination of the three models. We also do not know the exact shape of the field line close to the central engine, and so we cannot reconstruct the initial acceleration which might have helped discriminate between the models, as suggested by the divergence of the poloidal and toroidal velocities observed in Fig. 18. Our result however strongly favors a moderate value of $\mu$ with an efficient transformation of Poynting flux to kinetic energy (Models 1 and 2).

\subsubsection{Transition to a kinetically dominated jet}

The wind solution obtained for the sheath suggests a total specific energy at the base of the jet $\mu \sim 6-10$. In the first two models described in Sect. 4.4.2, conversion between magnetic and kinetic energy is efficient, and the equipartition condition $\sigma \sim 1$ is achieved at:

$r_{\mathrm{eq}} \sim 1.5$ mas $\sim 10 r_{\mathrm{lc}} \sim 200 R_{\mathrm{s}}$,

corresponding to

$z_{\text {eq }} \sim 20$ mas $\sim 2800 R_{\mathrm{s}} \sim 1.6 \mathrm{pc}$.

Results from MHD simulations of AGN jets (Komissarov et al. 2007) indicate $r_{\mathrm{eq}} \sim 30 r_{\mathrm{lc}}$. Considering the choice of total energy $\mu \sim 18$ used for these simulations, our results for Model 1 and Model 2 are qualitatively similar to the results of Komissarov et al. (2007).

The conversion of Poynting flux to kinetic energy is less efficient for our Model 3, and equipartition is only achieved at $r_{\mathrm{eq}} \sim 40$ mas $\sim 300 r_{\mathrm{lc}}$. However this model is altogether less favorable as it does not reproduce the observed acceleration as well as the first two models.

\subsection{Nature of the spine}

In Sect. 4.1, we identified two primary velocity components in the jet. Further analysis has shown that the slow component reflects either pattern speed of a plasma instability or the bulk speed of an outer slower wind. The fast velocity component has been successfully associated with the bulk speed along a magnetic field line connected to the observed jet shape, and we have obtained a wind solution for it. An inner deboosted part of the jet could therefore be associated with an even faster speed along a field line located closer to the jet axis.

The limb brightened structure found in the M 87 jet essentially at all scales might be explained by the combination of an enhanced magnetic field at the jet edge, a faster speed close to the jet axis and a lower emissivity in the jet spine. At kiloparsec scales, kinematic analysis systematically found a higher apparent velocity in the optical domain (Biretta et al. 1999; Meyer et al. 2013) compared to the radio domain (Biretta et al. 1995; Cheung et al. 2007; Giroletti et al. 2012) ${ }^{2}$, indicating that they trace different regions in the jet, and that the inner part (spine) of the jet is dominant at optical wavelength, as was also suggested earlier by Perlman et al. (1999).

In the stacked-epoch image of the jet in M 87, we measure an intensity ratio between the inner part (the spine) and the edge (the sheath) $\mathcal{R} \sim 0.5$ at $z_{\text {obs }} \sim 3$ mas. At this distance, we also observe $\gamma_{\text {sheath }} \sim 2.7$. Assuming that the lower intensity near the jet axis is mainly due to deboosting of the spine, we can calculate an estimate of the Lorentz factor of the spine necessary to reproduce the observed intensity ratio using:

$\mathcal{R}=\frac{I_{\text {spine }}}{I_{\text {sheath }}}=\left[\frac{\gamma_{\text {sheath }}\left(1-\beta_{\text {sheath }} \cos (\theta)\right)}{\gamma_{\text {spine }}\left(1-\beta_{\text {spine }} \cos (\theta)\right)}\right]^{2-\alpha}$.

Solving this equation, we obtain $\gamma_{\text {spine }} \sim 6$ 6.5. If we combine this estimate with the speed observed at HST-1 in the optical and assume that they are connected to a single field line, we can find a wind solution that would satisfy these observations. To obtain an accurate estimate of the velocity at HST-1 in the optical, we take the weighted mean of the four apparent velocities measured by Biretta et al. (1999) at HST-1, which gives us $\gamma_{\text {spine }}=9.8 \pm 1.9$.

Starting with the best solution found in Sect. 4.4.2 using Model 1, we set $\Omega=2.5 \times 10^{-6} \mathrm{~s}^{-1}$ corresponding to a field line anchored to a launching location closer to the ISCO $\left(r_{0} \sim\right.$ $\left.2.8 R_{\mathrm{s}}\right), \mu=12$, and $A=6.6$ so that $r_{\text {spine }}(z)=0.5 r_{\text {sheath }}(z)$. We then look for a valid wind solution that would fit the two estimates of the Lorentz factor of the jet spine. The result, plotted in Fig. 19, indicates that while the sheath field line can be associated with the velocity measured in the radio at HST-1, the spine field line connects well the inferred velocity of the deboosted structure in $43 \mathrm{GHz}$ VLBA maps and the velocity measured at HST-1 in the optical band.

While this model would explain the limb brightened structure at $z_{\text {obs }} \gtrsim 2$ mas, the decrease in spine speed close to the core would result in an accompanying increase in Doppler boosting of the spine. In the simplest interpretation a ridge brightened structure at $z_{\mathrm{obs}} \sim 1$ mas with brightness ratio $\mathcal{R} \sim 5$ would occur. This is not observed in the $7 \mathrm{~mm}$ VLBA observations, or in the 3 mm Global VLBI Array (GMVA) maps (Hada et al. 2016). This lack of ridge brightening suggest a low spine emissivity as the reason for the observed limb brightening down to the smallest core separations.

\footnotetext{
2 Giroletti et al. (2012) also discussed a potential faster spine component, and later observations of this region by Hada et al. (2014) constrained the estimated speed of this component to $\sim 5 c$.
} 


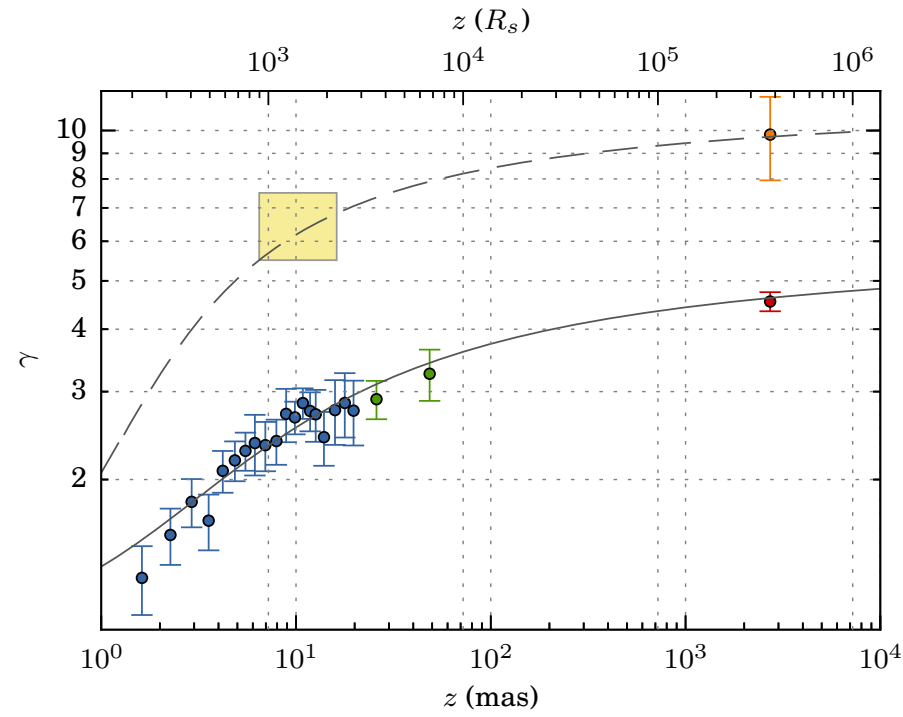

Fig. 19. Wind solution for the spine (dashed line) and the sheath (solid line). The solution for the spine fits well the inferred velocity of the deboosted structure observed in the $43 \mathrm{GHz}$ VLBA maps (yellow box) and the velocity observed at HST-1 in the optical band (orange point). The solution for the sheath fits the fast velocities measured in the $43 \mathrm{GHz}$ (blue points) and $15 \mathrm{GHz}$ (green points) VLBA data, and at HST-1 in the radio band (red point). The $15 \mathrm{GHz}$ VLBA data points are binned averages of the fastest observed apparent velocities from our WISE analysis.

\subsection{Launching mechanism}

The angular velocity of a sheath field line has been obtained independently from the observed flow rotation and from the modeling of jet acceleration and collimation. The two estimates agree remarkably well, within the uncertainty, with value $\Omega \sim$ $10^{-6} \mathrm{~s}^{-1}$. This parameter can be used to discriminate between the two major mechanisms that operate during jet launching. In the Blandford-Payne model, the jet is launched from the accretion disk and the field line angular velocity depends on the distance from the black hole at which this field line is anchored. If we assume a Keplerian rotation in the accretion disk, this angular velocity corresponds to $r_{0} \sim 5 R_{\mathrm{s}}$. In the Blandford-Znajek mechanism, the angular velocity depends on the spin of the central black hole $a_{\mathrm{H}}$. Following Tchekhovskoy (2015), the black hole angular speed is:

$$
\begin{aligned}
\Omega_{\mathrm{H}} & =\frac{a_{\mathrm{H}} c}{2 r_{\mathrm{H}}} \\
r_{\mathrm{H}} & =\frac{R_{\mathrm{s}}}{2}\left(1+\sqrt{1-a_{\mathrm{H}}^{2}}\right) .
\end{aligned}
$$

One can then obtain the angular speed of the respective field line, $\Omega_{\mathrm{F}} \simeq 0.5 \Omega_{\mathrm{H}}$.

Using $230 \mathrm{GHz}$ VLBI observations, Doeleman et al. (2012) estimated the spin of the black hole in M 87 to be $a_{\mathrm{H}} \sim 0.6$ based on a measure of the size of the smallest resolvable structure, identified as the ISCO. This value corresponds to $\Omega_{\mathrm{F}}=2.75 \times 10^{-6} \mathrm{~s}^{-1}$ and is a factor three times larger than the angular velocity that we determined. The BP mechanism would therefore better account for our result, but a BZ launching cannot be firmly discarded owing to the uncertainty of this measurement. Nevertheless, the wind solutions obtained for the sheath and the spine imply $\mu_{\text {spine }} \gtrsim \mu_{\text {sheath }}$, which is a characteristic of differential rotation as found both analytically and from numerical simulations (Lyubarsky 2009; Komissarov et al. 2007). Thus we favor a disk launching at least for the sheath.

\subsection{Mass-loss rate estimate}

The mass-loss rate corresponds to the amount of matter extracted from the accretion disk and loaded into the jet. It can be obtained from:

$\dot{M}=\frac{\eta \Psi_{0}}{4 c}$.

The wind solution describing the acceleration of the sheath requires $B_{0} / \eta \sim 2-10$, with $B_{0}$ describing the characteristic magnetic field at the light cylinder. The mass loss rate can then be estimated recalling that $\Psi_{0} \sim B_{0} r_{\mathrm{lc}}^{2}$ :

$\dot{M} \sim \frac{\eta B_{0} r_{\mathrm{lc}}^{2}}{4 c}$.

Using $230 \mathrm{GHz}$ VLBI observations, Kino et al. (2015) estimated the magnetic field at the base of the flow to be about 100 Gauss. Using this value for $B_{0}$, we get $\dot{M} \sim 10^{-7}-10^{-8} M_{\odot} \mathrm{yr}^{-1}$.

The mass accreted onto the black hole of M 87 is still undetermined. Using Chandra X-ray observations, a Bondi accretion rate of $\dot{M}_{\mathrm{B}}=0.12 M_{\odot} \mathrm{yr}^{-1}$ was estimated by Di Matteo et al. (2003). However, the measured X-ray luminosity, $L_{X} \sim 7 \times$ $10^{40} \mathrm{erg} \mathrm{s}^{-1}$, suggests a mass-loss rate of $\dot{M}_{\text {acc }} \ll \dot{M}_{\mathrm{B}}$. Kuo et al. (2014) found an upper limit of $\dot{M}_{\text {acc }}<1 \times 10^{-3} M_{\odot} \mathrm{yr}^{-1}$ using Faraday rotation. This limit would yield $\dot{M} \sim 10^{-4}-10^{-5} \dot{M}_{\text {acc }}$.

Thus, the mass-loss rate is much lower than the total mass accreted onto the black hole, implying that only a small fraction of the accreted matter is transported into the jet.

\section{Summary}

In this paper, we have presented a detailed analysis of the two-dimensional kinematic evolution of the innermost part of the jet in M 87. This source has allowed us to investigate jet formation and propagation on scales of $10^{2}-10^{3} R_{\mathrm{s}}$. A WISE analysis (Mertens \& Lobanov 2015, 2016) was performed on $43 \mathrm{GHz}$ VLBA maps observed as part of the M 87 VLBA movie project (Walker et al. 2008; in prep.). We summarize here the main findings of this analysis:

1. We have obtained the first complete velocity field of an AGN jet at sub-parsec scale, revealing a structured and highly stratified jet, with a significant transverse velocity component.

2. The structure of the flow was also investigated using SWD decomposition. The shape of the streamline is parabolic, well described by a power law $r \propto z^{0.6}$.

3. We observe an oscillation in flow expansion with a spatial period increasing with the distance as $\sim z_{\text {obs }}$, which is correlated with a similar oscillating pattern in the apparent speed.

4. The viewing angle $\theta \simeq 18^{\circ}$ was obtained using the speed of a moving component discovered in the counter jet, and the jet to counter jet intensity ratio. This viewing angle was also confirmed by the analysis of jet rotation and by the modeling of jet acceleration.

5. Flow stratification was analyzed using the SCC method (Mertens \& Lobanov 2016), revealing a slow, mildly relativistic layer $(\beta \sim 0.5 c)$ associated either with an outer wind or with instability pattern speed, and a fast accelerating streamline $\left(\gamma \sim 2.5\right.$ at $z_{\mathrm{obs}} \sim 3$ mas $)$ 
6. Acceleration and collimation of the flow was modeled as an MHD jet, using two different methods. We found that the acceleration is at first linear $\gamma \propto r \propto z^{0.58}$, and then saturates, implying a total specific energy $\mu \sim 6$, and that equipartition between Poynting flux and kinetic energy is reached at a distance $z_{\text {eq }} \sim 3000 R_{\mathrm{s}}$, in excellent agreement with previous analytic and numerical simulation work. This suggests that the jet is already kinetically dominated at a distance of a few parsecs from the central engine.

7. We could not detect any features that could unequivocally be related to an inner spine. We estimated a spine Lorentz factor $\gamma_{\text {spine }} \sim 6$ that would deboost the spine emission sufficiently to explain the intensity ratio between sheath and spine. Subsequently we found a wind solution that would connect this Lorentz factor with the velocity observed at knot HST-1 in the optical regime. However, this solution breaks down close to the core where it would predict a ridge brightened instead of a limb brightened jet. This suggests a lower intrinsic emissivity for the jet spine.

8. Indication of jet rotation was found from the difference in velocities observed in the northern and southern limbs. This observation was used to obtain an angular velocity for the field line, $\Omega \sim 10^{-6} \mathrm{~s}^{-1}$ which, in turn, suggests that this streamline is most likely launched in the inner region of the accretion disk, at a distance $r_{0} \sim 5 R_{\mathrm{s}}$ from the central engine. However, a BZ scenario in which the jet is launched from the magnetosphere of the central engine could not be excluded.

9. Finally, an estimate of the mass-loss $\dot{M} \sim 10^{-7}-10^{-8} M_{\odot} \mathrm{yr}^{-1}$ was obtained for the sheath, from the wind solution. This corresponds to $\dot{M} \sim 10^{-4}-10^{-5} \dot{M}_{\text {acc }}$.

Taken together, the WISE analysis of the jet in M 87 draws a coherent picture of a magnetically launched, accelerated, and collimated jet. Continuation and expansion of the work presented in this paper will enable testing the results obtained for the jet in M 87 and addressing other important aspects of the physics of relativistic flows. The methods that we have used to model the acceleration and collimation zone in the M 87 jet can be improved further to provide a more detailed physical description of the flow. One possibility would be to use a more realistic flux function obtained from numerical simulations. Another possibility would be to attempt to reproduce our results by performing full 2D or 3D MHD simulations using the physical parameters estimated for the jet in M 87. This would allow us to investigate the inner part of the jet, providing further clues about the reason for the edge brightened morphology.

The milliarcsecond-scale jet in M 87 has been observed at $15 \mathrm{GHz}$ as part of the MOJAVE project. We demonstrated in this work that the fast stream line can be robustly detected at this frequency with VLBA observations made roughly every two months. Obtaining and analysing a well-sampled $15 \mathrm{GHz}$ VLBA dataset on M 87 would enable us to extend the analysis of the acceleration profile up to $z \sim 10^{4} R_{\mathrm{s}}$. The high resolution provided by the GMVA observations at $86 \mathrm{GHz}$ could also be critical in confirming the results we obtained. The structure of the flow could be probed with GMVA at distances as small as $\sim 10 R_{\mathrm{s}}$, providing an excellent probe of the innermost part of the jet.

Acknowledgements. This research has made use of data from the MOJAVE database that is maintained by the MOJAVE team (Lister et al. 2009). F.M. was supported for this research through a stipend from the International Max Planck Research School (IMPRS) for Astronomy and Astrophysics at the Universities of Bonn and Cologne. We sincerely thank Y. Kovalev for providing the VLBA images at $2 \mathrm{~cm}$. F.M. thanks Y. E. Lyubarsky, C. Fendt, K. Toma and A. Tchekhovskoy for helpful discussions. We would also like to thanks the anonymous referee for the careful review that improved the quality of this paper. The National Radio Astronomy Observatory is a facility of the National Science Foundation operated under cooperative agreement by Associated Universities, Inc.

\section{References}

Agudo, I., Bach, U., Krichbaum, T. P., et al. 2007, A\&A, 476, L17 Agudo, I., Marscher, A. P., Jorstad, S. G., et al. 2012, ApJ, 747, 63 Anderson, J. M., Li, Z.-Y., Krasnopolsky, R., \& Blandford, R. D. 2003, ApJ, 590, L107

Asada, K., \& Nakamura, M. 2012, ApJ, 745, L28

Asada, K., Nakamura, M., Doi, A., Nagai, H., \& Inoue, M. 2014, ApJ, 781, L2 Begelman, M. C., \& Li, Z.-Y. 1994, ApJ, 426, 269

Biretta, J. A., \& Junor, W. 1995, Proc. Nat. Acad. Sci., 92, 11364

Biretta, J. A., Zhou, F., \& Owen, F. N. 1995, ApJ, 447, 582

Biretta, J. A., Sparks, W. B., \& Macchetto, F. 1999, ApJ, 520, 621

Blandford, R. D., \& Znajek, R. L. 1977, MNRAS, 179, 433

Blandford, R. D., \& Payne, D. G. 1982, MNRAS, 199, 883

Bodo, G., Rosner, R., Ferrari, A., \& Knobloch, E. 1996, ApJ, 470, 797

Camenzind, M. 1986, A\&A, 156, 137

Cheung, C. C., Harris, D. E., \& Stawarz, L. 2007, ApJ, 663, L65

Choi, M., Kang, M., \& Tatematsu, K. 2011, ApJ, 728, L34

Cohen, M. H., Meier, D. L., Arshakian, T. G., et al. 2015, ApJ, 803, 3

Daly, R. A., \& Marscher, A. P. 1988, ApJ, 334, 539

Di Matteo, T., Allen, S. W., Fabian, A. C., Wilson, A. S., \& Young, A. J. 2003, ApJ, 582, 133

Dodson, R., Edwards, P. G., \& Hirabayashi, H. 2006, PASJ, 58, 243

Doeleman, S. S., Fish, V. L., Schenck, D. E., et al. 2012, Science, 338, 355

Fendt, C. 2011, ApJ, 737, 43

Fendt, C., \& Ouyed, R. 2004, ApJ, 608, 378

Gebhardt, K., Adams, J., Richstone, D., et al. 2011, ApJ, 729, 119

Giroletti, M., Hada, K., Giovannini, G., et al. 2012, A\&A, 538, L10

Hada, K., Doi, A., Kino, M., et al. 2011, Nature, 477, 185

Hada, K., Kino, M., Doi, A., et al. 2013, ApJ, 775, 70

Hada, K., Giroletti, M., Giovannini, G., et al. 2014, in Proc. 12th European VLBI Network Symposium and Users Meeting (EVN 2014), 18

Hada, K., Kino, M., Doi, A., et al. 2016, ApJ, 817, 131

Hardee, P. E. 2000, ApJ, 533, 176

Hardee, P. E., \& Eilek, J. A. 2011, ApJ, 735, 61

Harms, R. J., Ford, H. C., Tsvetanov, Z. I., et al. 1994, ApJ, 435, L35

Homan, D. C., Lister, M. L., Kovalev, Y. Y., et al. 2015, ApJ, 798, 134

Hovatta, T., Aller, M. F., Aller, H. D., et al. 2014, AJ, 147, 143

Kino, M., Takahara, F., Hada, K., et al. 2015, ApJ, 803, 30

Komissarov, S. S., Barkov, M. V., Vlahakis, N., \& Königl, A. 2007, MNRAS, 380,51

Komissarov, S. S., Porth, O., \& Lyutikov, M. 2015, Comput. Astrophys. Cosmol., 2,9

Kovalev, Y. Y., Lister, M. L., Homan, D. C., \& Kellermann, K. I. 2007, ApJ, 668, L27

Kuo, C. Y., Asada, K., Rao, R., et al. 2014, ApJ, 783, L33

Lee, S.-S., Lobanov, A. P., Krichbaum, T. P., et al. 2008, AJ, 136, 159

Lee, S.-S., Lobanov, A. P., Krichbaum, T. P., \& Zensus, J. A. 2016, ApJ, 826, 135

Li, Z.-Y., Chiueh, T., \& Begelman, M. C. 1992, ApJ, 394, 459

Lister, M. L., Cohen, M. H., Homan, D. C., et al. 2009, AJ, 138, 1874

Lister, M. L., Aller, M. F., Aller, H. D., et al. 2013, AJ, 146, 120

Lobanov, A. P. 1998, A\&A, 330, 79

Lobanov, A. P., \& Zensus, J. A. 1996, in Energy Transport in Radio Galaxies and Quasars, eds. P. E. Hardee, A. H. Bridle, \& J. A. Zensus, ASP Conf. Ser., 100,109

Lobanov, A. P., \& Zensus, J. A. 1999, ApJ, 521, 509

Lobanov, A. P., \& Zensus, J. A. 2001, Science, 294, 128

Lobanov, A. P., Hardee, P. E., \& Eilek, J. A. 2003, New Astron. Rev., 47, 629

Ly, C., Walker, R. C., \& Junor, W. 2007, ApJ, 660, 200

Lyubarsky, Y. E. 2009, ApJ, 698, 1570

Lyubarsky, Y. E. 2010, MNRAS, 402, 353

Marshall, H. L., Miller, B. P., Davis, D. S., et al. 2002, ApJ, 564, 683

Mei, S., Blakeslee, J. P., Côté, P., et al. 2007, ApJ, 655, 144

Meier, D. L., Koide, S., \& Uchida, Y. 2001, Science, 291, 84

Mertens, F., \& Lobanov, A. P. 2015, A\&A, 574, A67

Mertens, F., \& Lobanov, A. P. 2016, A\&A, 587, A52

Meyer, E. T., Sparks, W. B., Biretta, J. A., et al. 2013, ApJ, 774, L21 
F. Mertens et al.: Kinematics of the jet in M 87 on scales of 100-1000 Schwarzschild radii

Norman, M. L., Winkler, K.-H. A., Smarr, L., \& Smith, M. D. 1982, A\&A, 113, 285

Owen, F. N., Hardee, P. E., \& Cornwell, T. J. 1989, ApJ, 340, 698

Perlman, E. S., Biretta, J. A., Zhou, F., Sparks, W. B., \& Macchetto, F. D. 1999, AJ, 117, 2185

Porth, O. 2013, MNRAS, 429, 2482

Reid, M. J., Biretta, J. A., Junor, W., Muxlow, T. W. B., \& Spencer, R. E. 1989 , ApJ, 336, 112

Sol, H., Pelletier, G., \& Asseo, E. 1989, MNRAS, 237, 411

Staff, J. E., Koning, N., Ouyed, R., Thompson, A., \& Pudritz, R. E. 2015, MNRAS, 446, 3975

Tchekhovskoy, A. 2015, in The Formation and Disruption of Black Hole Jets, eds. I. Contopoulos, D. Gabuzda, \& N. Kylafis, Astrophys. Space Sci. Libr., 414,45
Tchekhovskoy, A., McKinney, J. C., \& Narayan, R. 2008, MNRAS, 388, 551 Toma, K., \& Takahara, F. 2013, Progr. Theor. Exper. Phys., 2013, $083 E 02$ Tsinganos, K., \& Bogovalov, S. 2002, MNRAS, 337, 553

Vlahakis, N. 2015, in The Formation and Disruption of Black Hole Jets, eds. I. Contopoulos, D. Gabuzda, \& N. Kylafis, Astrophys. Space Sci. Libr., 414, 177

Vlahakis, N., \& Königl, A. 2004, ApJ, 605, 656

Walker, R. C., Ly, C., Junor, W., \& Hardee, P. J. 2008, J. Phys. Conf. Ser., 131, 012053

Walker, R. C., Ly, C., Junor, W., \& Hardee, P. 2009, in Approaching MicroArcsecond Resolution with VSOP-2: Astrophysics and Technologies, eds. Y. Hagiwara, E. Fomalont, M. Tsuboi, \& M. Yasuhiro, ASP Conf. Ser., 402, 227

Walsh, J. L., Barth, A. J., Ho, L. C., \& Sarzi, M. 2013, ApJ, 770, 86 


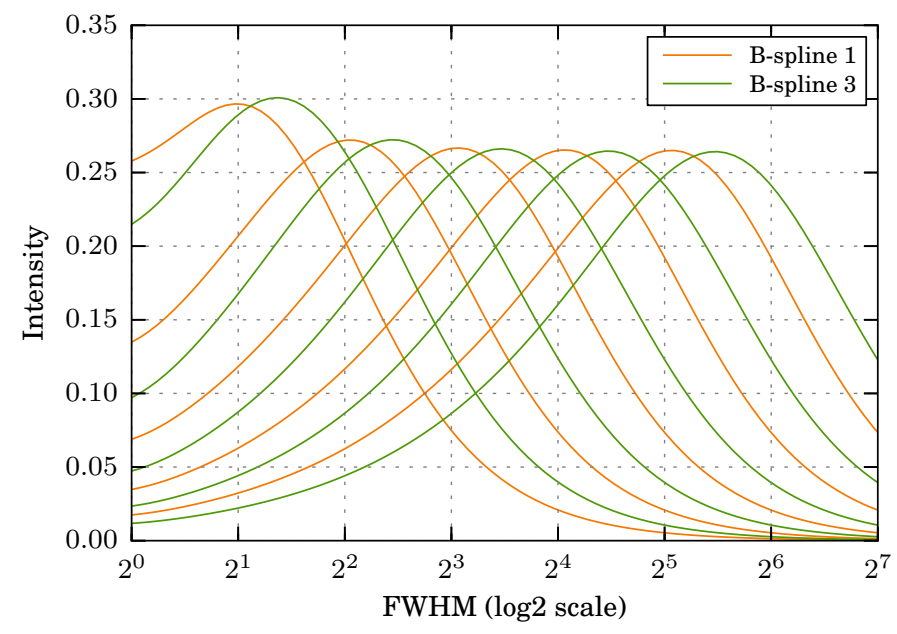

Fig. A.1. Relation between scales of a wavelet transform and spatial scales expressed in units of the FWHM of a Gaussian PSF of an image. The individual curves represent the relative sensitivity of a given wavelet scale to recovering a Gaussian feature with a given FWHM. The FWHM values for which the individual wavelet scales reach their maximum sensitivity are marked in the abscissa. The combination of two B-spline functions is used in the IWD algorithm to provide the enhanced sensitivity over a refined range of spatial scales.

\section{Appendix A: Intermediate-scales wavelet decomposition}

Canonical wavelet transformations are performed on a set of scales ranging as powers of two of the initial, smallest scale used. The ability of the multiscale cross correlation (MCC; Mertens \& Lobanov 2015) procedure to cross identify significant structural patterns (SSPs) in pairs of images relies on a smooth transition between scale $j$ and scale $j+1$ of the wavelet transform employed. This condition sets limits on detecting different speeds at different scales, and hence providing an accurate assessment of flow stratification traced by overlapping optically thin features with different intrinsic speeds (Mertens \& Lobanov 2016). For any two adjacent scales of the wavelet transform, this limit is on the order of $2^{j}$ (Mertens \& Lobanov 2015). As the velocity difference between two overlapping features becomes comparable or smaller than this limit, matching becomes more difficult. To improve cross identification of the individual features, one can construct a finer scaling function for which the wavelet decomposition will cover intermediate scales. This is performed by introducing an intermediate-scale wavelet decomposition (IWD). In a canonical wavelet transform, the coefficients $w_{j}$ at a scale $j$ of the wavelet decomposition contains information on spacial scales between $2^{j-1}$ and $2^{j}$. In the IWD, the coefficients at a given scale $j$ contain information on spacial scales between $1.5 \times 2^{j-1}$ and $1.5 \times 2^{j}$. The MCC is then performed by inserting the scale $j$ of the IWD between the scales $j-1$ and scale $j$ of the SWD.

The IWD is realized in the WISE analysis by introducing an appropriate scaling function. The $B$-spline scaling function used in WISE has the following discrete low pass filter associated with it:

$$
\left(\begin{array}{lll}
1 / 4 & 1 / 2 & 1 / 4
\end{array}\right)
$$

For the IWD, a modified $B$-spline 3 scaling function is used which has a discrete low pass filter in the following form:

$$
\left(\begin{array}{lllll}
1 / 16 & 1 / 4 & 3 / 8 & 1 / 4 & 1 / 16
\end{array}\right)
$$

The resulting sensitivity of the IWD to different structural scales is illustrated in Fig. A.1 which compares the responses of different wavelet scales to different spatial scales in the image, for the scaling functions $B$-spline and $B$-spline 3. 